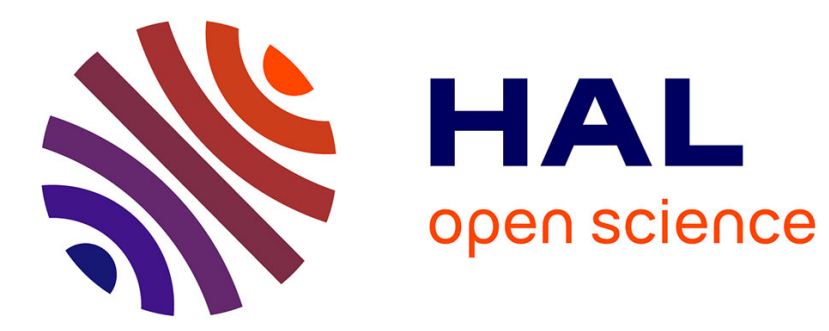

\title{
Deterministic particle transport in a ratchet flow
}

Philippe Beltrame, Mounia Makhoul, Maminirina Joelson

\section{To cite this version:}

Philippe Beltrame, Mounia Makhoul, Maminirina Joelson. Deterministic particle transport in a ratchet flow. Physical Review E: Statistical, Nonlinear, and Soft Matter Physics, 2016,93 (1), 24 p. 10.1103/PhysRevE.93.012208 . hal-01270218

\section{HAL Id: hal-01270218 \\ https://hal.science/hal-01270218}

Submitted on 5 Feb 2016

HAL is a multi-disciplinary open access archive for the deposit and dissemination of scientific research documents, whether they are published or not. The documents may come from teaching and research institutions in France or abroad, or from public or private research centers.
L'archive ouverte pluridisciplinaire HAL, est destinée au dépôt et à la diffusion de documents scientifiques de niveau recherche, publiés ou non, émanant des établissements d'enseignement et de recherche français ou étrangers, des laboratoires publics ou privés. 


\section{Deterministic particle transport in a ratchet flow}

ARTICLE in PHYSICAL REVIEW E · JANUARY 2016

Impact Factor: 2.29

READS

13

3 AUTHORS:

\section{Philippe Beltrame}

Université d'Avignon et des Pays du Vaucluse 25 PUBLICATIONS 92 CITATIONS

SEE PROFILE

\section{Joelson Maminirina}

Université d 'Avignon et des Pays du Vaucluse

53 PUBLICATIONS

120 CITATIONS

\section{Mounia Makhoul}

Université d'Avignon et des Pays du Vaucluse 5 PUBLICATIONS 0 CITATIONS

SEE PROFILE

SEE PROFILE 


\title{
Deterministic particle transport in a ratchet flow
}

\author{
Philippe Beltrame, ${ }^{*}$ Mounia Makhoul, and Maminirina Joelson \\ Université d'Avignon et des Pays de Vaucluse-INRA, UMR1114 EMMAH, F-84914 Avignon, France \\ (Received 21 November 2013; revised manuscript received 14 December 2015; published 14 January 2016)
}

\begin{abstract}
This study is motivated by the issue of the pumping of particle through a periodic modulated channel. We focus on a simplified deterministic model of small inertia particles within the Stokes flow framework that we call "ratchet flow." A path-following method is employed in the parameter space in order to retrace the scenario which from bounded periodic solutions leads to particle transport. Depending on whether the magnitude of the particle drag is moderate or large, two main transport mechanisms are identified in which the role of the parity symmetry of the flow differs. For large drag, transport is induced by flow asymmetry, while for moderate drag, since the full transport solution bifurcation structure already exists for symmetric settings, flow asymmetry only makes the transport effective. We analyzed the scenarios of current reversals for each mechanism as well as the role of synchronization. In particular we show that, for large drag, the particle drift is similar to phase slip in a synchronization problem.
\end{abstract}

DOI: 10.1103/PhysRevE.93.012208

\section{INTRODUCTION}

Microfluidic devices devoted to particle sorting have been extensively developed since the late 20th century [1-5] with applications ranging from biological to chemical and industrial fields. Particle transport along narrow channels with periodic properties is a feature of many devices reported in the literature [6]. Such a system allows a mass dependent sorting process as demonstrated in the experimental setup in Refs. [7,8]. The promising results of the experiment presented in Ref. [8] motivated the present work. In that experiment, a pore lattice, built in a macroporous silicon wafer, was connected at both ends to basins. The basins and the pores were filled with liquid and micrometer-sized particles. Sinusoidal pumping drags the particles. The experiment showed the existence of an effective transport in specific ranges of parameter values and in addition the possibility to invert the transport direction by varying the pumping frequency. Among the many mechanisms which may explain these results, Refs. [8,9] interpret the slow particle drift as a ratchet effect called drift ratchet [9-11]. "Ratchet effect" refers to the possibility of transporting particles even if the mean force is zero (zero bias) or, more surprisingly, in the opposite direction to the bias. In the drift ratchet, the authors of the cited references propose a minimal model in which inertia is neglected and the particle advects with the flow under thermal fluctuations. Such a model of an overdamped ratchet under Brownian noise has been widely used to explain transport in many field of physics, and reviews can be found in Refs. [12] or [10]. However, a recent experiment on particle transport in pores [13] revealed that thermal fluctuations are negligible and that the Stokes drag is the dominating force acting on the particles.

In the present paper, we address a complementary problem: Is it possible to achieve this kind of transport for larger particles when noise is neglected and small inertia is taken into account? We therefore seek deterministic mechanisms responsible for the transport. Among the deterministic transport mechanisms of suspended particles with zero-mean force, the Stokes drift

\footnotetext{
*philippe.beltrame@univ-avignon.fr
}

is an important factor that we found in many frameworks especially in sediment transport [14], fluid mixing processes [15], and Langmuir circulation in the ocean [16,17]. However, the drift is due to a nonzero average of the traveling wave motion along a Lagrangian trajectory. Therefore, there is fluid transport. Yet we seek a particle drift without fluid transport. In this way, the flow needs to be ratchetlike. A common way to classify ratchets is by the kind of potential and forcing [10]. For example, when the potential depends only on space and the external force is time periodic, it is called a rocking ratchet, whereas when the potential depends on space and time, as for the Stokes drift, it is called a pulsating ratchet. The present study employs a kind of fluctuating potential ratchet where the potential is the product of the spatial and temporal variations, i.e., a standing wave.

The generation of a current in a ratchet can in a statistical sense be understood in a unified point of view as an out-of-equilibrium phenomenon within the framework of a symmetry analysis [18-21]. In one-dimensional systems, two symmetries have to be broken, one related to spatial parity symmetry and the other to time-reversal symmetry. For instance, inertia breaks time-reversal symmetry. The definition of these symmetries will be detailed in Sec. II. Note that these symmetry conditions are necessary but not sufficient to induce transport and even if the latter exists, it provides no information about the kind of particle dynamics.

The deterministic approach raises questions about the existence of unbounded particle trajectories which may exist without net current, since even for the one-dimensional problem, the dynamics of deterministic inertia ratchets is quite complex and regions of chaotic behavior are ubiquitous. For certain parameter values, the chaotic dynamics disappears suddenly and a regular transport takes place. The transition was identified as a crisis and as a synchronization phenomenon in the early 2000s [22,23]. Owing to the $L$-spatial and the $T$-temporal periodicities, the velocity is rational, i.e., $c=m L / n T$, where $m$ and $n$ are integers and the velocity $c$ remains locked to this value in its existence domain. In the literature (e.g., Ref. [24]), this is referred to as phase locking or phase synchronization since the dynamics is synchronized with the periodic forcing. Such a synchronization is not specific 
to the ratchet problem but is rather a generic phenomenon of periodically forced nonlinear oscillators [25,26]. Another noteworthy property of dynamics in ratchet is current reversal corresponding to a change in the transport direction by varying a bifurcation parameter such as the pumping frequency. In the early 2000s different scenarios were reported using time integration. Mateos [22,27] linked current reversal with chaosto-order transition and evoked tangent bifurcation. In contrast, Barbi and Salerno [23] showed the existence of a current reversal without chaotic dynamics but rather the coexistence of different periodic attractors. In Ref. [24] a more general interpretation of current reversal was presented starting with the symmetric case. In this case, the existence of one transport solution implies the existence of the opposite one for a symmetric initial condition. If the symmetry is slightly broken, e.g., by a constant bias, the stability domains of the two opposite solutions no longer match. Then, by varying the bifurcation parameter, a current reversal may be observed. The relevance of the coexistence of transport solutions in opposite directions was corroborated more recently [28].

Other groups have pointed out the role of symmetry in current reversal $[21,29,30]$. However, its role is fundamentally different: asymmetry determines the direction of the transport. The current reversal therefore results from a change of asymmetry. In this framework, Ref. [31] proved a very generic result for deterministic or stochastic rocking ratchets, showing that the transport velocity is a function of phase shift between the different harmonics of the rocked force. This general result may explain many current reversals with biharmonic forcing or asymmetric potential. However, the main assumption is that the transport velocity depends only on the rocked force and not on the initial conditions. Therefore, these results cannot be applied when current multiplicity exists as in Ref. [24].

Although a plethora of studies have focused on inertia ratchets, little is known about ratchets with weak particle inertia. Usually the inertia is assumed to be large or at least of order one [22-24,27]. The case of small inertia is addressed as the limiting case of an overdamped particle under stochastic fluctuations $[9,32]$. One of the goals of the present paper was to find the order of magnitude of inertia needed to obtain transport. The second goal concerns the emergence of unbounded dynamics and transport. The transitions reported in the literature focus on the synchronization threshold of transport solutions. However, the transition from bounded to unbounded dynamics does not seem to be clearly identified. This question is especially relevant for our case since, for small enough inertia, the dynamics is bounded. Last, we investigated whether symmetry may play different roles in the existence of a transport solution: is it either a spontaneous symmetry breaking or induced only by asymmetry, as for a mechanical pawl?

In order to answer these questions, we propose to use numerical bifurcation analysis (see Ref. [33] for a review) in addition to direct time simulation. The advantage of this method is its ability to follow periodic orbits independently of their stability and to find their domain of existence. Such a skeleton of the dynamics has proven effective to find the onset of transport and to understand the transition. The continuation method is seldom used for the simple ratchet problem, but paradoxically it is used in a more complex situation with particle interaction in a narrow channel [6]. In particular, there the authors highlight the coexistence of several transport solutions which are hard to detect with direct time integration.

In the present work, we focus on an axisymmetric model in which a single particle moves along the channel axis and its motion results only from the competition between fluid driving and small inertia. This model is called ratchet flow. The main parameters are the relative drag (inverse of the inertia), the characteristic flow velocity related to the pressure difference in the experiment, and the shape of the flow velocity related to the pore shape (Sec. II). The last is characterized by the velocity contrast and the asymmetry parameter which breaks the parity transformation $x \rightarrow-x$. We first analyze the transport solutions due to the spontaneous symmetry-breaking mechanisms of these solutions (symmetric case Sec. III A). Second, we treat the case of asymmetry as a perturbation of the previous case since it appears crucial to understand the current reversal (Sec. III B). In Sec. IV we seek another transport solution which only exists because of the forced parity symmetry breaking. Finally, we interpret the transitions as synchronization phenomena (Sec. V), and concluding remarks are addressed in Sec. VI.

\section{MODEL AND BASICS}

\section{A. Governing equation of the particle}

Let us consider a $L$-periodically modulated channel infinitely extended along the line $(O x)$ through which a viscous fluid and a suspended particle are sinusoidally pumped. We call "pore" the channel portion of length $L$ (Fig. 1). Following the drift ratchet study in Ref. [9], the fluid motion can be assumed quasistatic. More specifically, for a characteristic velocity $u_{m}$ about $2-10 \mathrm{~mm} / \mathrm{s}$, a pumping frequency about $40-100 \mathrm{~Hz}$ and characteristic length related to the minimum pore radius $r_{\min }$ about $1-10 \mu \mathrm{m}$ (see Fig. 1), the Reynolds number $\operatorname{Re}=\frac{r_{\min } u_{m}}{v}$ varies in the range $2 \times 10^{-3}-10^{-1}$ and the product $\mathrm{Re} S t=\frac{R_{\min }^{2}}{v T}$ varies in the range $2.5 \times 10^{-4}-10^{-2}(\mathrm{St}$ is the Strouhal number). Therefore, the convective and local accelerations are negligible compared to the viscous stress leading to the so-called Stokes flow. The validity of the Stokes approximation $\Delta \vec{v}=\nabla p$ was corroborated by using the full nonlinear Navier-Stokes equation (see Ref. [34]). Then, under the creeping flow approximation, the time dependence of the velocity field is governed by the sinusoidal pumping, and it takes the form $v(\vec{r}, t)=v(\vec{r}) \sin 2 \pi t$. We assume that the problem is axisymmetric: the particle is centered on the $x$ axis. Then, the drag force on the particle is along the $x$ only, and we obtain a one-dimensional dynamics of a particle of

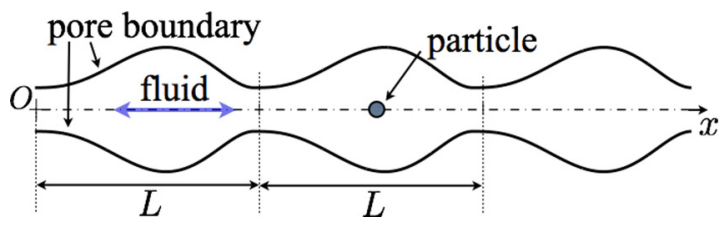

FIG. 1. Sketch of the problem: the particle moves along the $x$ axis of a $L$-periodically modulated channel. Each channel element of length $L$ constitutes a "pore." The periodic motion of the viscous fluid drags the particle. 
mass $m$ according to the Newton's second law

$$
m \ddot{x} \mathbf{e}_{\mathbf{x}}=R(x, t) \mathbf{e}_{\mathbf{x}},
$$

where $R$ is the resultant force when the effect of gravity is neglected. In a confined domain the pore boundary has a non-negligible influence and except for a cylindrical domain, there are no analytical results [35]. Recently, using a boundary element approach, we computed this force in the Stokes approximation framework [36,37]. Here we do not focus on the numerical method, but we relate this force to the particle velocity and other parameters.

Because pressure and viscous stress depend linearly on the boundary condition, the particle drag force $R$ can be written as the sum of two contributions [36]:

$$
R=F_{p}+F_{r}
$$

The first contribution $F_{p}$ corresponds to the drag force on a moving particle in a fluid at rest while the second term $F_{r}$ is the drag force on a particle at rest in a moving fluid. Again because of the linearity argument, $F_{p}$ is proportional to the particle velocity $V_{p}$ so we can write $F_{p}=-\gamma V_{p}$ with $\gamma>0$ the drag coefficient. $F_{r}$ is proportional to the pressure difference amplitude; we therefore introduce the velocity $u_{0}$, called the equivalent velocity field, such that $F_{r}=\gamma u_{0} \sin (2 \pi t / T)$. In the limiting case of a point particle in an infinite medium $u_{0}$ corresponds to the velocity of the fluid without the particle, and then $R$ is the Stokes drag. For the general case of a fluid confined in a micropore with a non-negligible particle size, $u_{0}$ depends on the pore boundary, and the particle shape as explained in Ref. [37]. Hence, for an axisymmetric problem the equivalent velocity field $u_{0}(x)$ is a function of the position $x$ of the particle. Likewise, the drag coefficient depends on the pore boundary and thus on the $x$ position [38]. Therefore, the drag force on a particle centered at $x$ with the velocity $V_{p}=\dot{x}$ (the dot designates the time derivative) is expressed as

$$
\mathbf{R}=-\gamma(x)\left[\dot{x}-u_{0}(x) \sin (2 \pi t / T)\right] \mathbf{e}_{\mathbf{x}} .
$$

The equation (1) of motion of the particle is nonlinear because of the functions $u_{0}(x)$ and $\gamma(x)$. The computation of these coefficients is detailed in Refs. [36,39] for different pore shapes and particle sizes. It is shown that for rather small particles, the relative variation of $\gamma(x)$ is negligible compared to that of the equivalent velocity $u_{0}(x)$. Thus, in this paper we assume that $\gamma$ is constant not only to simplify the problem but also to point out the case where the nonlinearity is only due to $u_{0}(x)$ : it is called the ratchet flow. If the length is scaled by the pore length $L$, the time by the pumping period $T$ and the drag by $m / T$, then the dimensionless problem is written

$$
\ddot{x}+\gamma \dot{x}=\gamma u_{0}(x) \sin (2 \pi t) .
$$

This equation admits a unique solution $C^{2}$ for a given position and velocity $\left(x_{i}, v_{i}, t_{i}\right)$ at a time $t_{i}$. In particular, two different solutions cannot have at a given time the same position and velocity. Another straightforward result shows that particle acceleration $\ddot{x}$ and its velocity $\dot{x}$ remain bounded. Note that the driving flow [right-hand side in Eq. (4)] corresponds to a standing wave. The situation is therefore different from the Stokes drift, which involves a traveling wave of the driving flow.
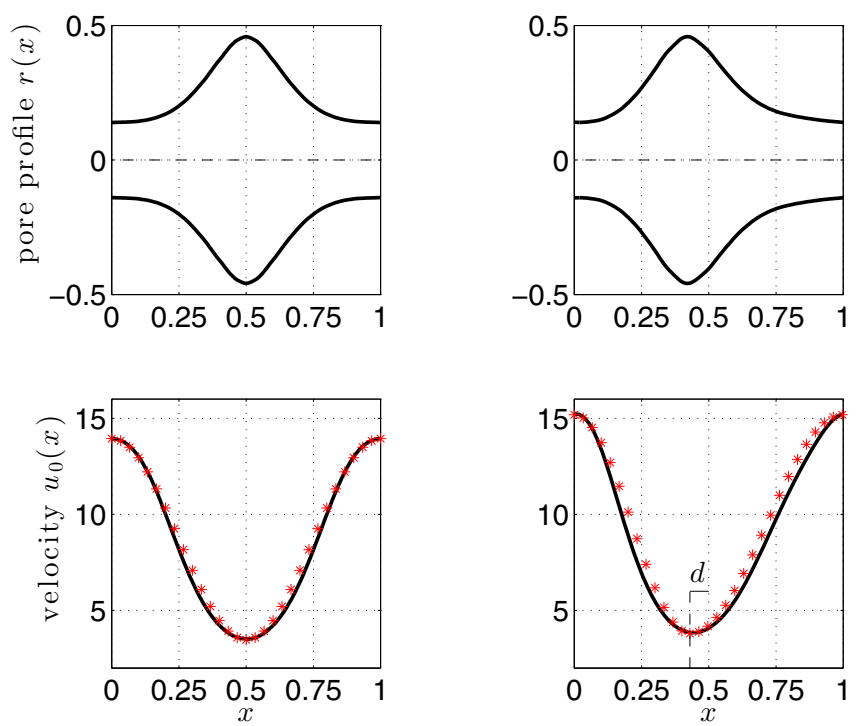

FIG. 2. Examples of the velocity field $u_{0}(x)$ for the symmetric (left panel) and the asymmetric (right panel) pore profiles. The plain lines in the bottom panels correspond to the velocity profiles computed using the algorithm developed in Ref. [36]. The red stars in the left and right panels are the analytical fields for $a=0.6$ of Eqs. (10) and (5), respectively. The parameter $d$ is the algebraic difference between $x=0.5$ and the velocity minimum. To plot the analytical profile in the right panel, we use $d=-0.07$. Note also that the velocity extrema of the asymmetric case are slightly shifted w.r.t. the extrema of the pore profiles.

\section{B. Velocity field $u_{0}(x)$}

We propose to construct an analytical velocity field $u_{0}(x)$ qualitatively similar to the one computed in Ref. [37] and characterized by three parameters: the mean value $u_{m}$, the asymmetry noted by $d$, and the velocity contrast or relative amplitude $a$. According to Ref. [37], for the symmetric case, $u_{0}(x)$ is maximal at the bottleneck while it is minimal at the largest pore radius. More generally, the number of velocity extrema are determined by those of the pore radius. Figure 2 shows that $u_{0}$ has one maximum and one minimum and when the pore is asymmetric, the velocity minimum is slightly shifted relatively to the middle position $x=1 / 2$ (Fig. 2). For the pore profiles of Fig. 2, the $u_{0}$ field can be approximated analytically by

$$
\begin{aligned}
u_{0}\left(x, u_{m}, a, d\right)= & u_{m}\left(1+a\left\{\cos \left(\frac{\pi \bar{x}}{d+1 / 2}\right) \mathbb{1}_{[0 ; 1 / 2+d]}(\bar{x})\right.\right. \\
& \left.\left.+\cos \left[\frac{\pi(\bar{x}-1)}{d-1 / 2}\right] \mathbb{1}_{]^{1 / 2+d ; 1]}}(\bar{x})\right\}\right),
\end{aligned}
$$

where $d$ is the algebraic shift, which ranges from $-1 / 2$ to $1 / 2$, $\bar{x}=x \bmod 1$, and $\mathbb{1}_{I}$ is the indicator function of the interval $I\left[\mathbb{1}_{I}(\bar{x})=1\right.$ if $\bar{x} \in I$, otherwise $\left.\mathbb{1}_{I}(\bar{x})=0\right]$. This equation also applies to the symmetric case $(d=0)$ where $u_{0}(x, a, 0)$ becomes the sinusoidal function

$$
u_{0}\left(x, u_{m}, a, 0\right)=u_{m}[1+a \cos (2 \pi x)] .
$$

The advantage of this formulation compared to the usual biharmonic profile as in Ref. [9] is, first, that it avoids two maxima of the velocity field which do not occur in our pore 
geometry and, second, that the asymmetry of the velocity profile can be controlled by tuning the parameter $d$. This parameter turns out to be crucial for the transport analyzed in Sec. IV since its sign determines the transport direction. Even though $d$ may approach $1 / 2$, the computation of $u_{0}$ shows that we cannot reach this limit value. In fact, $d$ is typically about 0.1 . Additionally, a large velocity gradient is not consistent with the Stokes approximation. Concretely, we do not consider values of $d$ above to 0.4 .

Without loss of generality, the study can be restricted to positive values of $d$, since we have the following equality: $u_{0}\left(x, u_{m}, a,-d\right)=u_{0}\left(-x, u_{m}, a, d\right)$. Then if a transport solution exists for $d>0$, it exists in the opposite direction for $-d<0$.

\section{Continuation method}

In addition to time integration, we employ the so-called continuation or path-following method applied to periodic solution branches. Their bifurcations and stability will help to understand a possible transition with the transport solutions as performed by Ref. [6]. We need to interpret the ODE (4) as a dynamical system of the variable $s(t)=(x(t), v(t))$ :

$$
\dot{s}=F(s, t)=\left\{\begin{array}{l}
v \\
\gamma u_{0}\left(x, u_{m}, a, d\right) \sin 2 \pi t-\gamma \dot{v}
\end{array}\right.
$$

We used the freeware package AUTO [40] which requires an autonomous system. Sinusoidal forcing can be done by adding a nonlinear oscillator with the desired forcing as one of the solution components [41]. The nonlinear oscillator has to converge and to be asymptotically stable in order to avoid numerical problems. Then we add two equations to the dynamical system Eq. (7):

$$
\begin{gathered}
\dot{\sigma}=2 \pi \varsigma+\sigma\left(1-\varsigma^{2}-\sigma^{2}\right), \\
\dot{\zeta}=-2 \pi \sigma+\varsigma\left(1-\varsigma^{2}-\sigma^{2}\right),
\end{gathered}
$$

and we replace $\sin 2 \pi t$ in Eq. (7) by $\sigma(t)$ since it converges asymptotically to $\sin (2 \pi t+\phi)$ and $\varsigma$ to $\cos (2 \pi t+\phi)$ with $\phi$ a constant phase [42]. Therefore Eq. (7) coupled with the two-dimensional autonomous system Eq. (8) have the same limit cycles. A periodic solution $x(t)$ is a periodic orbit $s$ in the phase space of the ODE Eq. (8). It is computed by solving the associated boundary value problem as explained in Refs. [40,43]. The stability of the solution is determined using Floquet multipliers. Then, by varying the system parameters, we are able to track the branches of periodic orbits and determine their stability. Moreover it is possible to detect bifurcations, for instance, pitchfork bifurcations, period doubling, or saddle node, which is also called fold bifurcation for periodic orbits [44]. They are denoted in the bifurcation diagrams by the letters "PB," "PD," and "LP," respectively. Each point on the bifurcation diagrams represents the norm $\|$. I of a $T$-periodic solution $s$. Its norm is defined as the $L_{2}$-norm of the particle velocity:

$$
\|s\|=\left[\frac{1}{T} \int_{0}^{T} v^{2}(t) d t\right]^{1 / 2} .
$$

Then the norm does not depend on the particle position, and thus two identical motions shifted by a spatial period have the same norm.

\section{The periodic solutions $s_{m}$ and $s_{0}$}

There are no general analytical results for the nonlinear equation (4). Nevertheless, for small velocity $u_{m}$, the particle trajectory during a pumping period is small compared to the pore length. Under this assumption, the case is analogous to that of the drift of charged particles under ponderomotive force, i.e., in a nonuniform oscillating electromagnetic field $[45,46]$ where particles collect either at minima or maxima of the field. In our case, we prove in Appendix A the existence of a pair of saddle limit cycles with period one. The stable solution is centered at the minimum of the velocity $u_{0}, x=1 / 2+d$, and it is denoted $s_{m}$. The second unstable solution is centered at the maximum of the velocity, $x=0$, and it is denoted $s_{0}$. The resulting dynamics is, after a transient drift, a periodic motion of the $s_{m}$ solution.

\section{E. Symmetry and consequence}

The role of symmetry, other than (space and time) shift symmetry, in the ratchet effect has been discussed in the literature $[20,21]$. The existence of a transport solution requires that the space and time reflection symmetries be broken. The existence of a nonzero inertia already breaks the time-reversal symmetry called supersymmetry in Ref. [20]. If the pore geometry has the parity symmetry $x \rightarrow-x \quad(d=0)$, an effective transport is not possible. However, this fact does not forbid the existence of transport solutions for a given initial condition. Any possible transport solution is then associated to a symmetric copy in the opposite direction. The motivation for considering a parity symmetric geometry lies in a better understanding of the different mechanisms resulting in a ratchet effect. Indeed, we show in Sec. III that the existence of a transport solution is related to spontaneous symmetry-breaking bifurcations. Moreover, the asymmetric case can be interpreted as a perturbation of the symmetric case what allows to state in Sec. III B that the multiplicity of solutions for a slightly asymmetric profile is not a coincidence but is due to the symmetry. An additional consequence is the existence of current reversal scenarios.

The symmetric case corresponds to the parity symmetry of the velocity $u_{0}$, which occurs for $d=0$ :

$$
u_{0}\left(-x, u_{m}, a, 0\right)=u_{0}\left(x, u_{m}, a, 0\right) .
$$

Thus, the evolution problem Eq. (7) is equivariant under the spatiotemporal parity symmetry $\mathcal{S}$ :

$$
F(\mathcal{S}(s, t))=-F(s, t), \text { with } \mathcal{S}(s, t)=(-s, t+1 / 2) .
$$

The symmetry $\mathcal{S}$ is obtained by a central symmetry in the $(x, v)$ plane followed by a time shift of a half period. The equivariance property implies that for any trajectory $x(t)$ of Eq. (7), the dynamics $-x(t+1 / 2)$ is a solution too. Note that the parity symmetry of $u_{0}$ [Eq. (10)] implies the existence of another symmetry $\mathcal{S}_{1 / 2}$ centered on $x=1 / 2$ instead of 0 . Indeed, $\mathcal{S}$ and $\mathcal{S}_{1 / 2}$ define the same symmetry if we introduce the relative solution $\bar{s}=(\bar{x}, v) \in S^{1} \times \mathbb{R}$ where the relative 
position is defined modulo one: $\bar{x}=x \bmod 1$. In this context, a solution $(\bar{S}, t)$ is "symmetric" if it is an invariant of $\mathcal{S}$ in $S^{1} \times \mathbb{R} \times \mathbb{R}:$

$$
\mathcal{S}(\bar{s}, t)=(\bar{s}, t) \Longleftrightarrow \forall t: \bar{x}(t+1 / 2)=-\bar{x}(t) .
$$

A symmetric solution has the following properties: (1) it is 1-periodic, i.e., synchronized with the pumping, and (2) its mean position over one period $\langle\bar{x}\rangle$ is either 0 or $1 / 2$. The first property ensues from writing Eq. (12) as $x(t+1 / 2)=-x(t)+$ $n$, where $n \in \mathbb{Z}$. Since $x(t)$ is continuous, then $n$ has to be constant. Then we have $x(t+1)=-x(t+1 / 2)+n$ too. From these two equalities, we deduce the periodicity. The second property results from the time integration over one period of Eq. (12), and we get $\langle\bar{x}\rangle \equiv-\langle\bar{x}\rangle \bmod 1$. This equation has two solutions: $\langle\bar{x}\rangle=0$ or $1 / 2$. Hence there are two types of symmetric solutions, one centered at the pore inlet $(\bar{x}=0)$, which corresponds to the velocity maximum, and the second one at the middle position $\bar{x}=1 / 2$, which corresponds for the symmetric case to the velocity minimum. For $u_{m} \ll 1$, these two solutions are $s_{0}$ and $s_{m}$ found previously. Therefore, under this assumption $s_{0}$ is a saddle and $s_{m}$ is a node. Note that a corollary of these results is that an asymmetric solution has a mean that differs from 0 or $1 / 2$.

In order to obtain transport, one has to break the parity symmetry $\mathcal{S}$ of the dynamics either by spontaneous (Sec. III) or forced (Sec. IV) symmetry breaking.

\section{TRANSPORT VIA SPONTANEOUS SYMMETRY BREAKING}

In this section we focus on transport induced by spontaneous symmetry breaking. In other words we seek unbounded dynamics and transport in the symmetric case. In a second step, we analyze the effect of a break of the symmetry $(d \neq 0)$ on these dynamics.

\section{A. Symmetric case}

We assume that the problem is symmetric, i.e., $d=0$, and thus the system Eq. (7) is equivariant by the central symmetry $\mathcal{S}$. We depict, first, the bifurcations of periodic branches and especially their stability domain. Second, we identify the transitions from bounded dynamics to unbounded dynamics and from unbounded dynamics to periodic transport. The latter transport branches can be studied in the comoving frame in the same manner as for the bounded periodic solution. Finally, we point out how $n$-periodic solutions are related to such a periodic transport.

\section{Periodic solutions}

From the analytical results (Sec. II D), we identified two periodic symmetric solutions, $s_{0}$ and $s_{m}$, which are displayed in Fig. 3(a) $\left(\gamma=100, u_{m}=1\right.$, and $\left.a=0.65\right)$. As expected for small $u_{m}$, the particle converges to the only stable solution $s_{m}$ centered at $x=1 / 2$. The continuation of these solution branches with respect to the parameters $a$ or $\gamma$ does not display bifurcations and $s_{m}$ remains stable. Thus, all the dynamics is attracted by the only stable periodic solution $s_{m}$ when $u_{m}$ is small.
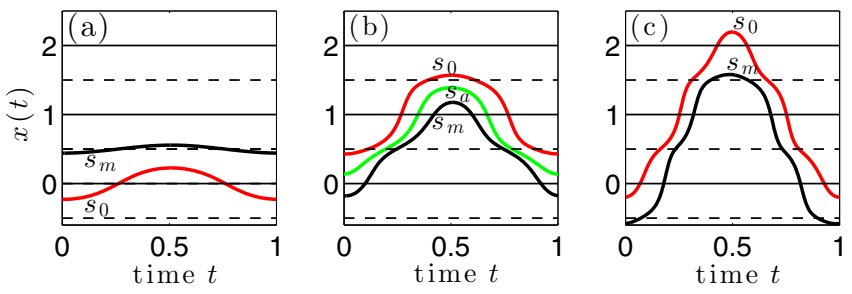

FIG. 3. Time evolutions of 1-periodic solutions (red) $s_{0}$, (black) $s_{m}$, and (green) $s_{a}$ for different values of $u_{m}$ with $\gamma=100, a=0.65$, and $d=0$ (symmetric case). (a) The two 1-periodic solutions (stable) $s_{0}$ and (unstable) $s_{m}$ for $u_{m}=1$. Panels (b) and (c) display solutions at the pitchfork bifurcations (PBs) in the bifurcation diagram (Fig. 4). Specifically, in (b) $s_{0}$ is the profile at $u_{m}=u_{p u}^{(1)}$ and $s_{m}$ is the profile at $u_{m}=u_{p \ell}^{(1)}$, and $s_{a}$ is the profile of the solution at $u_{m}=5.1$ of the branch $s_{a}$. In (c), $s_{0}$ is the profile at $u_{m}=u_{p \ell}^{(2)}$, and $s_{m}$ is the profile at $u_{m}=u_{p u}^{(2)}$.

Larger characteristic flow velocity $u_{m}$ has to be explored. So $u_{m}$ is set presently to be the bifurcation parameter while the other parameters are fixed, $a=0.65$ and $\gamma=100$. The related bifurcation diagram displays a snaking shape of the branches $s_{0}$ and $s_{m}$, which regularly cross each other (Fig. 4). Let us describe quantitatively the solution branches. When both branches are close to each other, they exchange their stability. The $s_{m}$ solution loses its stability at $u_{p \ell}^{(1)}=4.84$ while $s_{0}$ becomes stable at $u_{p u}^{(1)}=5.27$ (Fig. 4). A similar stability exchange occurs for $u_{m}$ about 9 . According to the time evolution plots in Figs. 3(b) and 3(c) of the different

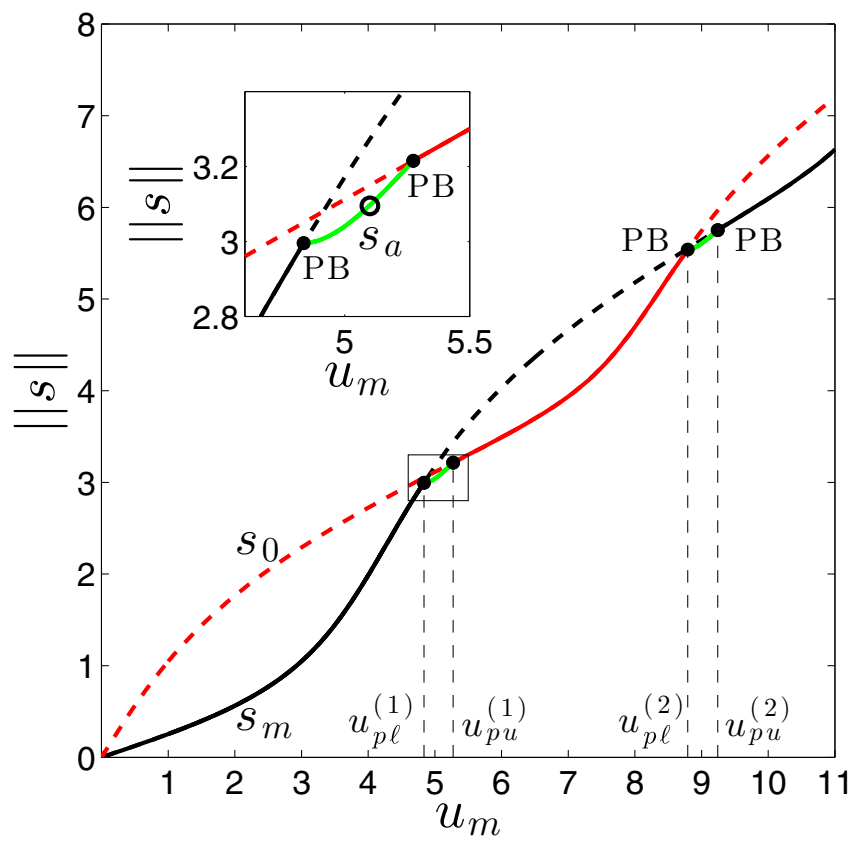

FIG. 4. Bifurcation diagram of period-one orbit branches as a function of the velocity contrast $a$ : (red line) $s_{0}$, (black line) $s_{m}$, and (green line) $s_{a}$. Plain (dashed) lines indicate stable (unstable) solution branches. Dots indicate pitchfork bifurcation points (PBs). Solution profiles located at the four pitchfork bifurcations and the $s_{a}$ profile indicated by the circle in the inset are shown in Figs. 3(b) and 3(c). Parameters are $\gamma=100, a=0.65$, and $d=0$ (symmetric case). 
solutions, their stability seems follow the present rule: if the peak of the trajectory reaches the region of the $u_{0}$ minimum (i.e., at $\bar{x}=1 / 2$ ), then the solution is stable, whereas if the trajectory reaches the $u_{0}$ maximum $(\bar{x}=0)$, then the solution is unstable. Therefore the stability changes when the oscillation amplitude increases roughly by a pore length, showing the relevance of the mean velocity $u_{m}$ for the stability of the solutions. The stability exchange implies the emergence of a new branch which breaks the $\mathcal{S}$ reflection symmetry via a pitchfork bifurcation (PB in Fig. 4). The bifurcated asymmetric branch is called $s_{a}$ [see Fig. 3(b)]. Indeed, there are two subbranches $s_{a+}$ and $s_{a_{-}}$, with solutions centered in the intervals ]0,1/2[ and ]1/2,1[ respectively. Because they are related to symmetry, $s_{a+}(t+1 / 2)=\mathcal{S}\left[s_{a-}(t)\right]$, they do not appear twice in the bifurcation diagram (Fig. 4). When the $s_{m}$ solution loses its stability at $u_{p \ell}^{(1)}$ the $s_{a}$ branch emerges supercritically and connects the branch $s_{0}$ at $u_{p u}^{(1)}$, which gains stability. The mean positions of the $s_{a}^{+}$and $s_{a}^{-}$solutions move from $1 / 2$ to 1 and to 0 , respectively. A similar scenario appears between the critical values $u_{p \ell}^{(2)}$ and $u_{p u}^{(2)}$ (Fig. 4). As the branches $s_{a}$ are stable, there is always a stable periodic solution. The time integration of Eq. (4) corroborates that dynamics is attracted by one of these 1-periodic solutions.

For smaller drag the $s_{a}$ branch can be unstable. In Fig. 5 we plot a similar bifurcation diagram to that in Fig. 4 with the same parameter values except $\gamma$, which is equal to 10 instead of 100. The bifurcation diagram displays a snakelike structure of the symmetric branches as previously. Such a structure is commonly encountered in the literature, e.g., the solution branches of droplets on a striped substrate $[47,48]$ or the localized state branches that are solutions of the SwiftHohenberg equation [49]. Moreover, the overall bifurcation diagram with the $s_{a}$ branch in Fig. 5 is very reminiscent of the tilted snakes-and-ladders structure encountered, e.g., in the conserved Swift-Hohenberg equation [50] in which the asymmetric branch $s_{a}$ is the rungs of the ladder and the snaking branches are the two symmetric branches. The common thread of all these diagrams is the spatial symmetry of the problem: shift and parity symmetries.

Each $s_{a}$ branch lets appear two period-doubling bifurcations denoted $u_{1 \ell}$ for the lower bifurcation and $u_{1 u}$ for the upper bifurcation of the $s_{a}$ branch in Fig. 5(b). Such a period-doubling bifurcation is generic for nonlinear oscillators with dissipation and additional time-dependent force [51]. Furthermore it can occur only with the asymmetric branch $s_{a}$ as is proven in Ref. [52] for bifurcation problems with the $\mathcal{S}$ symmetry. A sequence of period-doubling bifurcations is observed and leads to a temporally chaotic flow. The first period-doubling cascade starts at $u_{1 \ell}$ and the $2^{n}$-periodic branch emerges at the bifurcation point $u_{n \ell}$ [see Fig. 5(b)]. These points accumulate approximately at $u_{L} \simeq 8.23$ [Fig. 5(b)]. The present cascade has a behavior similar to that of one-dimensional maps where the ratio of distances between two consecutive bifurcations is the universal Feigenbaum constant $\delta \simeq 4.669$ [53,54]. Beyond this threshold a strange attractor is observed as expected. In the same manner, by decreasing $u_{m}$, the second period-doubling cascade occurs from $u_{1 u}$ [Fig. 5(b)]. The end of this cascade is hidden by the stable branch $s_{m}$, which emerges from the fold bifurcation at $u_{f s}$ [see Fig. 5(a)]. According to the selfsimilarity Feigenbaum law, the end of the cascade is estimated to be close to $u_{1 u}$ and smaller than $u_{f s}$. Thus, chaotic dynamics is observed for $u<u_{f s}$. In both cases the numerical simulation shows that the chaotic particle trajectory remains bounded in the vicinity of the thresholds. Other period-doubling cascades exist in the interval $\left[u_{L}, u_{f s}\right]$. Without detailing this complex scenario, we describe the first bifurcated branches which will be useful to understand the periodic transport.
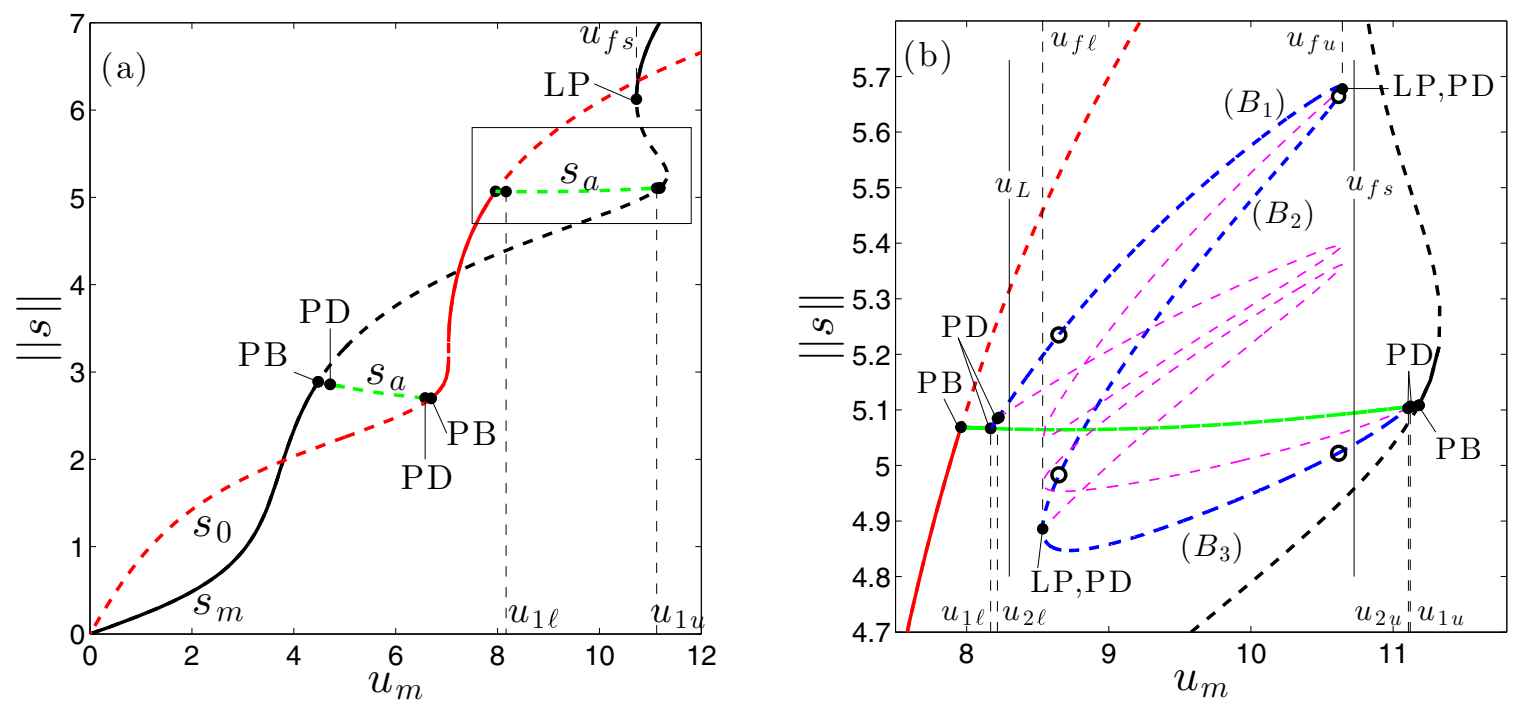

FIG. 5. (a) Bifurcation diagrams showing the periodic branches as a function of the characteristic velocity $u_{m}$ for $a=0.65$ and $\gamma=10$. (b) Magnification for $u_{m}$ ranging in [7; 12]. The color code for 1-periodic solutions is as in Fig. 4. The (dark blue) 2- and (magenta) 4-periodic branches emerge from the (green line) $s_{a}$ branch. Dots indicate the different bifurcations: Pitchfork bifurcation (PB), period doubling (PD), fold bifurcation (LP). In panel (b) the vertical line at $u_{L}$ corresponds to the end of the period-doubling cascade starting from $u_{1 \ell}$ with the emergence of chaotic dynamics. The circles on the $\left(B_{1}\right),\left(B_{2}\right)$, and $\left(B_{3}\right)$ branches correspond to solutions displayed in Figs. 6 and 15 . The critical values at the turning points are $u_{f \ell}=8.53431, u_{f u}=10.6433$. 

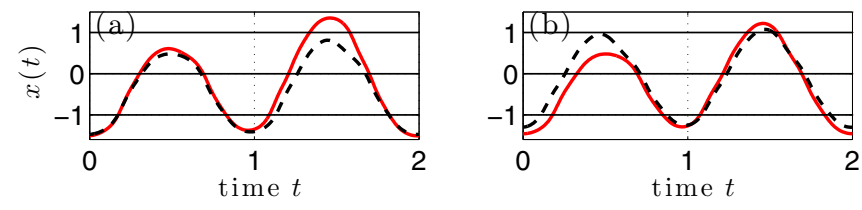

FIG. 6. Time evolutions of period-two orbits for two values of $u_{m}$ displayed by four circles in Fig. 5 : (a) $u_{m}=8.65$, (b) $u_{m}=10.62$. The plain red lines correspond to solutions on the branch $\left(B_{2}\right)$, whereas the dashed black lines correspond to (a) the circle on the $\left(B_{1}\right)$ branch and $(b)$ the circle on the $\left(B_{3}\right)$ branch.

Having two saddle nodes $u_{f \ell}$ and $u_{f u}$, the period-two branch defines three subbranches [Fig. 5(b)] which connect $\left(B_{1}\right)$ the period-doubling point $u_{1 \ell}$ to the first saddle node $u_{f u},\left(B_{2}\right)$ the two saddle nodes, and $\left(B_{3}\right)$ the $u_{f \ell}$ point to the upper period-doubling bifurcation $u_{1 u}$. Figure 6 shows that the trajectory of (B2) during the first period $(t \in[0 ; 1])$ may differ strongly from the trajectory during the second period $(t \in[1 ; 2])$, in particular the local maximum of the particle trajectory during the first period is significantly smaller than that of the trajectory during the second period. In contrast, for the same parameter values, the local maxima of the trajectories of $\left(B_{1}\right)$ and $\left(B_{3}\right)$ are only slightly different between the two periods (dashed line in Fig. 6). We will show in Sec. III A 4 that the existence of the $\left(B_{2}\right)$ branch plays a key role in the emergence of periodic transport. Note that the 2-periodic branch is not unstable on the whole range $\left[u_{L} ; u_{f s}\right]$. At the saddle-node bifurcation the solution regains stability, but in the neighborhood a 4-periodic branch emerges, and the period-two branch is unstable again. The relative difference between the saddle node, and these period-doubling bifurcations are less than $10^{-6}$ where stability "islands" appear. This scenario is also repeated by the 4-periodic branches [magenta in Fig. 5(b)] and the 8-periodic branches (not shown); thus each branch has stability islands in the neighborhood of the saddle nodes. Noteworthy, the turning points of the 2-, 4-, and 8-periodic branches occur at almost the same values. The direct time integration shows that it is certainly true for all the perioddoubled solutions. Therefore, apart from stability "islands," the $2^{n}$-periodic orbits $(n>1)$ are unstable.

In this instability region, unbounded dynamics has been found. In the next section we study the transition from bounded to unbounded dynamics.

\section{Unbounded attractor emergence}

Near the period-doubling cascade a bounded strange attractor emerges. Because of the shift symmetry, a series of attractors shifted by one pore length exists. The simulation shows that they are globally invariant w.r.t. the central symmetry $\mathcal{S}$. In other words, if $(\bar{x}, v)$ is a point of the attractor, then $(\overline{-x},-v)$ belongs to the same attractor. However, generically $\bar{x}(t+1 / 2) \neq-\bar{x}(t)$, and then the time periodicity is destroyed while the parity symmetry $(\bar{x}, v) \rightarrow(\overline{-x},-v)$ remains. This partial "symmetry restoring" is certainly due to a merging crisis between two copies of the asymmetric attractors as described in Ref. [55]. If we plot the Poincaré section of this strange attractor, we observe that its amplitude in the $x$ direction is inferior to the pore length. Increasing the

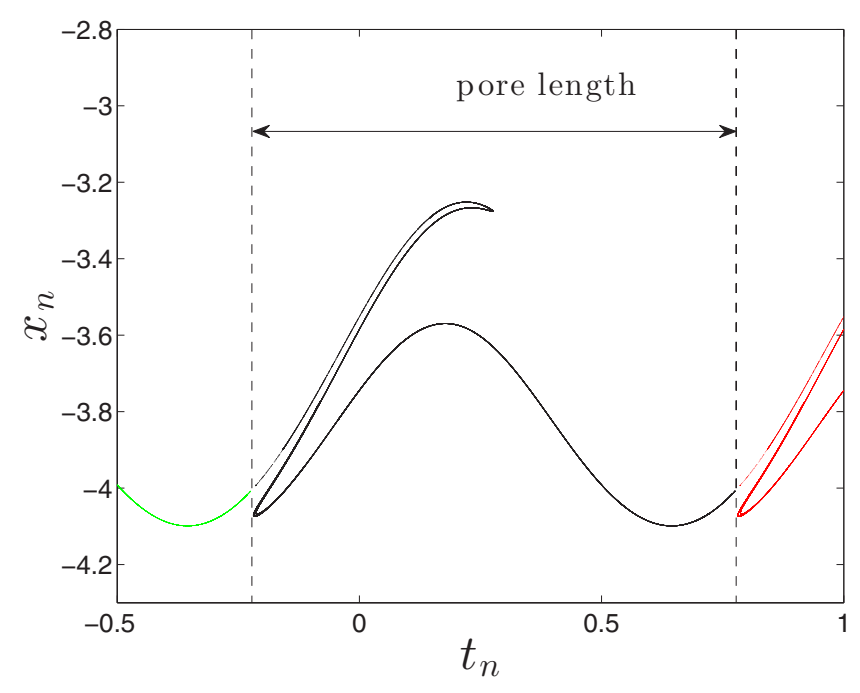

FIG. 7. Poincaré section $x_{n}=x(n), v_{n}=v(n)$ where $n \in \mathbb{N}$, near the onset of unbounded dynamics for $u_{m}=8.519$ (other parameters: $\gamma=10, a=0.65$, and $d=0$ ). (black line) The strange attractor between the dashed vertical lines is included in a pore length. (Colors) The shifted contiguous attractors are very close. Note that the Poincaré section does not reveal the central symmetry $\mathcal{S}$ of the strange attractor since the symmetric point appears for a temporal shift of a half period.

mean velocity $u_{m}$ the attractor expands until two contiguous attractors are close to each other (Fig. 7). At a critical value $u_{u B} \simeq 8.5198$ the attractors merge as for a merging crisis in systems with symmetry [55]. It follows that the dynamics is no longer bounded, and an intermittent dynamics takes place: the trajectories remain a long time near the "ghost" bounded attractor and switch rapidly to a contiguous attractor (Fig. 8). There is no net preference for a transition to the left or to the

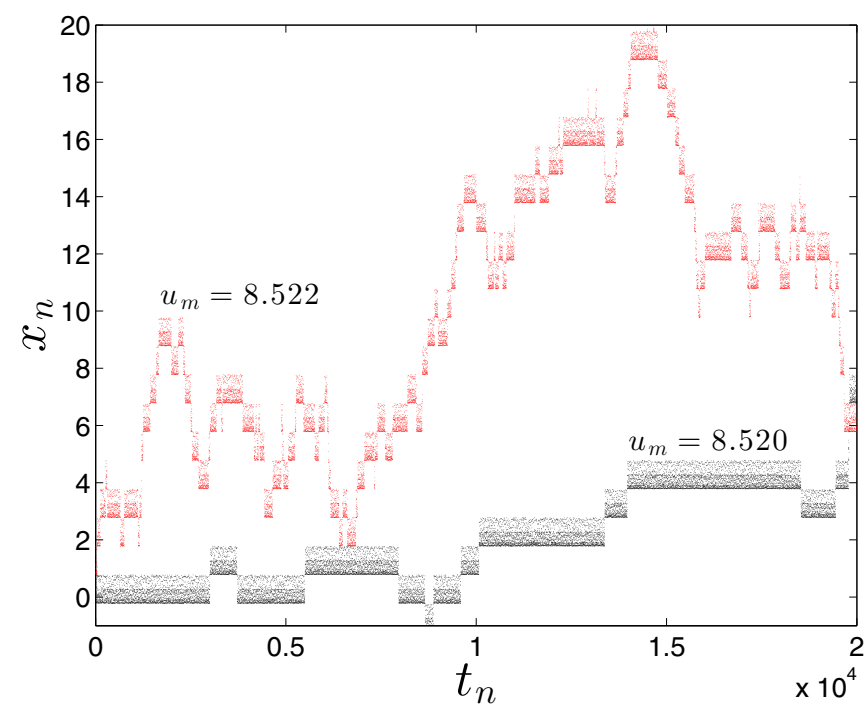

FIG. 8. Stroboscopic particle dynamics $x_{n}=x(n)$ at discrete time $t_{n}=n$ near the merging crisis for two different values of $u_{m}$ slightly superior to $u_{u B}$. Dynamics displays long chaotic bounded dynamics interrupted by jumps which shift the dynamics by one pore length. The velocity profile $u_{0}(x)$ is symmetric [Eq. (10)], $\gamma=100$ and $a=0.65$. 

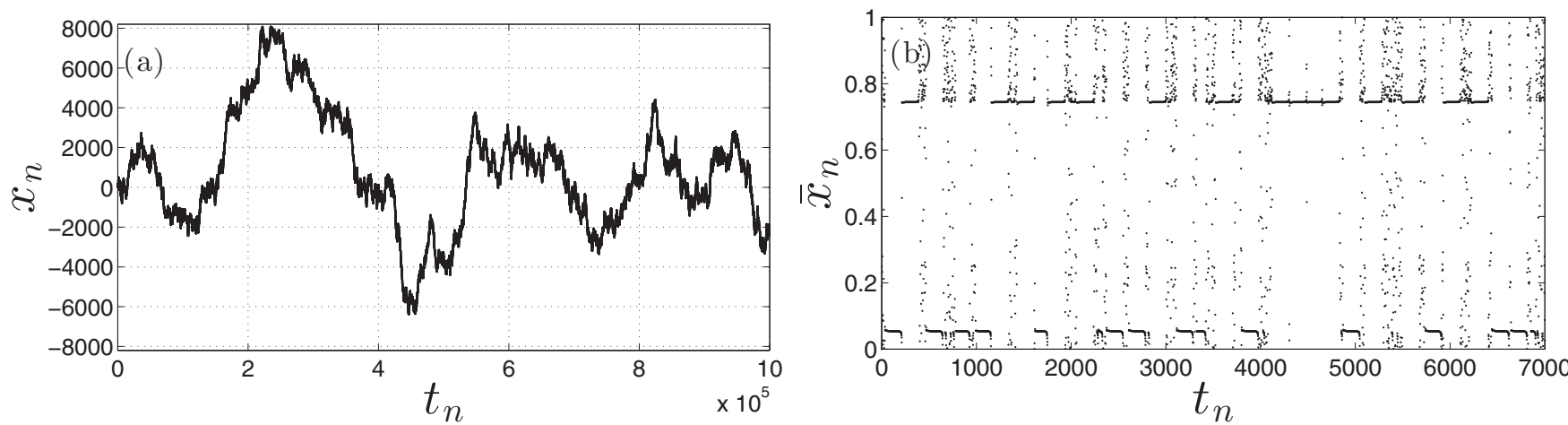

FIG. 9. Dynamics near the onset of periodic transport at $u_{m}=u_{f \ell}^{t}$. Shown is the (a) stroboscopic particle positions $x_{n}$ and the (b) relative stroboscopic particle positions $\bar{x}_{n}=x_{n} \bmod 1$. The dynamics in panel (a) is reminiscent of a random walk without preferential direction. However, the plateaux of panel (b) indicate a nearly periodic transport at the velocity $c$ equal to +1 or -1 . The duration of this regular transport is large (about a few hundred periods) compared to the chaotic dynamics (a few periods). Parameters: $u_{m}=8.64835, a=0.65, \gamma=10, d=0$.

right due to the symmetry of the bounded attractors involved in the transition. The duration of bounded dynamics epochs is irregular. Nevertheless, the mean duration decreases when $u_{m}$ moves away from the critical value.

On increasing the bifurcation parameter further, the transport epochs are longer than the bounded ones. Figure 9(a) presents alternating transport to the left and right with negligible epochs of bounded dynamics as in Fig. 8. Indeed, the transport events are nearly periodic as Fig. 9(b) shows. The duration of this regular dynamics increases when $u_{m}$ approaches $u_{f \ell}^{T} \simeq 8.64836$, which corresponds to the onset of the periodic transport solution studied in the next section. Starting from $u_{f u}$, a similar scenario occurs when decreasing $u_{m}$ (Fig. 5): at $u_{m} \simeq 10.65$, a transition from bounded chaotic to unbounded chaotic dynamics takes place, and it is followed by a regular transport at $u_{m}=u_{f u}^{T}=10.6169$. The bifurcation scenario starting from the periodic symmetric solutions and leading to synchronized transport is sketched in Fig. 10.

\section{Periodic transport solutions}

We study the regular transport emerging from the fold bifurcations $u_{f \ell}^{T}$ and $u_{f u}^{T}$. This transport is periodic in a comoving frame with the velocity $c=+1$ : After one temporal period, the particle moves one spatial period forward (Fig. 11). Thus the position $x_{T}^{+}(t)$ of the transport solution $s_{T}^{+}$is the sum of a period-one solution $x_{p}^{+}(t)$ and a uniform translation:

$$
x_{T}^{+}(t)=x_{p}^{+}(t)+t
$$

This transport solution is called periodic transport solution. Because of the $\mathcal{S}$-equivariance (11) of the problem, the existence of a transport solution $s_{T}^{+}$in the direction of the increasing $x$ (also called to the right) implies the existence of a

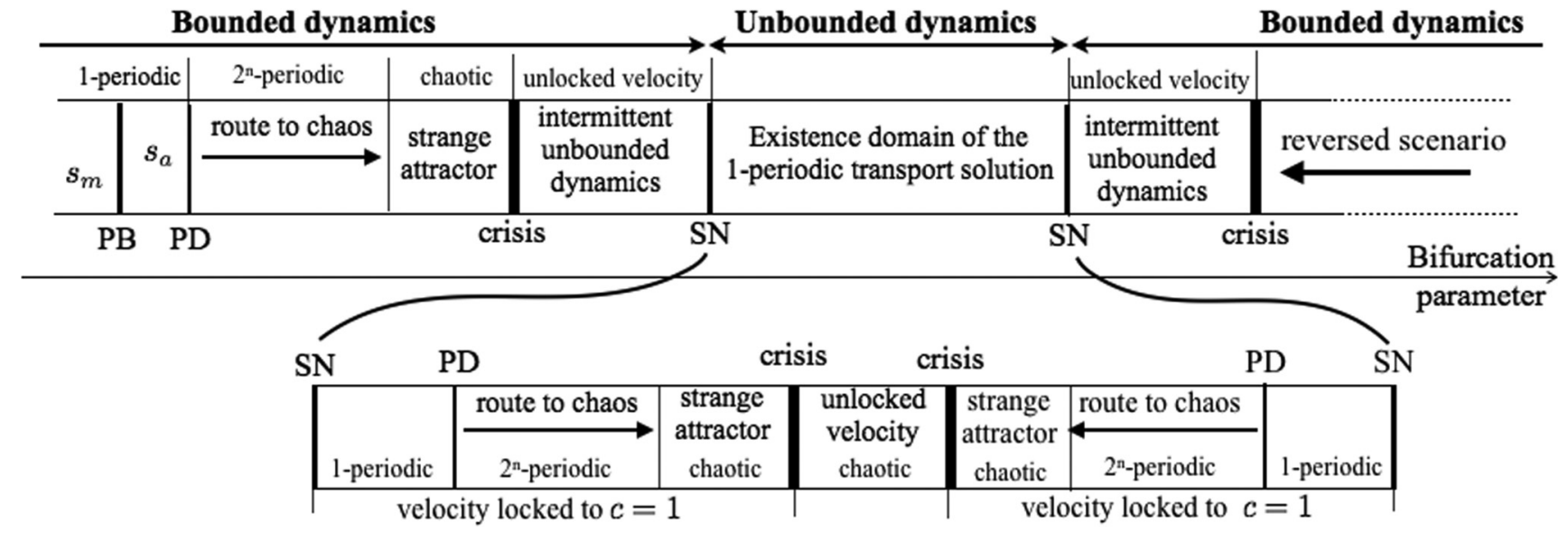

FIG. 10. Generic bifurcation scenarios encountered for moderate $\gamma \simeq 10$. (Top) Bifurcation scenario starting from symmetric 1-periodic solutions and leading to transport periodic solutions. More specifically: (i) Spatial and temporal symmetry breaking lead to the existence of strange attractors. (ii) A crisis allows for the birth of an unbounded intermittent dynamics. (iii) Finally the 1-periodic transport emerges at a saddle node. (Bottom) The 1-periodic transport solution loses its stability via a similar scenario of the bounded dynamics. However, until a widening crisis, the transport velocity remains locked to $c= \pm 1$. Beyond this crisis the velocity is no longer locked. An effective transport appears only for the asymmetric case. Increasing the bifurcation parameter further, we get again a synchronized transport by a similar reversed scenario. 


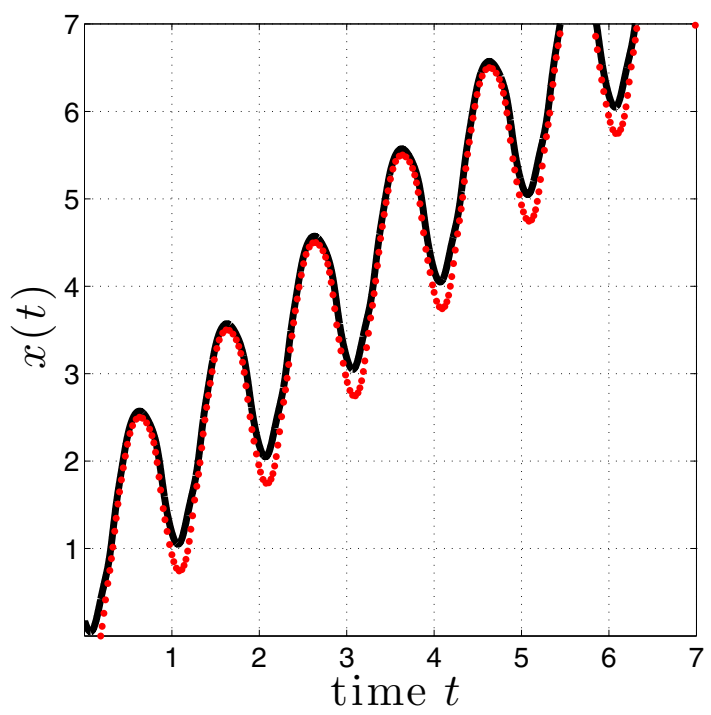

FIG. 11. Time evolution of the (plain) stable and (dotted) unstable transport solution $s_{T}^{+}$at $u_{m}=9$ (circles in the bifurcation diagram Fig. 12). After a temporal period, the particle advances by exactly one pore length.

symmetric transport solution $\left(s_{T}^{-}, t+1 / 2\right)=\mathcal{S}\left(s_{T}^{+}, t\right)$ for which the position $x_{T}^{-}$verifies the following relations:

$$
\begin{aligned}
x_{T}^{-}(t) & =-x_{T}^{+}(t+1 / 2) \\
& =-x_{p}^{+}(t+1 / 2)-1 / 2-t \\
& =x_{p}^{-}(t)-t .
\end{aligned}
$$

Then $s_{T}^{-}$is a period-one transport solution but in the opposite direction, i.e., to the left. For the symmetric case, one does not need to study the opposite transport solution, and the sign direction in the notation of the solution $s_{T}$ is dropped.

The continuation method is carried out within the periodic part $x_{p}$ of the transport solution, i.e., the transport solution $x_{T}$ in the comoving frame. If $x_{T}$ is a solution of the ODE (4), then $x_{p}$ is a solution of the new ODE:

$$
\ddot{x}_{p}+\gamma \dot{x}_{p}=\gamma\left[u_{0}\left(x_{p}+c t\right) \sin (2 \pi t)-c\right],
$$

where $c= \pm 1$ depends on the transport direction $s_{T}^{c}$. The presence of the constant bias $-c$ on the right side of Eq. (17) recalls the tilting force in ratchet problems [10]. This force tends to move the particle to the left if $c>0$. However, here we seek periodic solutions in the comoving frame, thus one aims at stopping the "natural" transport. In the comoving frame, this problem is analogous to the absolute negative mobility in tilted inertial ratchets (see, e.g., Ref. [56]).

The continuation of the periodic $x_{p}$ solution, with $u_{m}$ the bifurcation parameter, shows the existence of two branches $\left(T_{1}\right)$ and $\left(T_{2}\right)$ connecting the two folds $u_{f \ell}^{T}$ and $u_{f u}^{T}$ (Fig. 12). Then for each direction two transport solutions coexist in the same range. $\left(T_{1}\right)$ is always unstable while $\left(T_{2}\right)$ is stable at the emergence of the saddle nodes. This branch loses its stability via period doubling defining three stability ranges of $\left(T_{2}\right)$. The first range of stability starts from the lower fold and ends at the period-doubling bifurcation $u_{1 \ell}^{T 1}=8.68$, the second one is delimited by two period-doubling bifurcations at

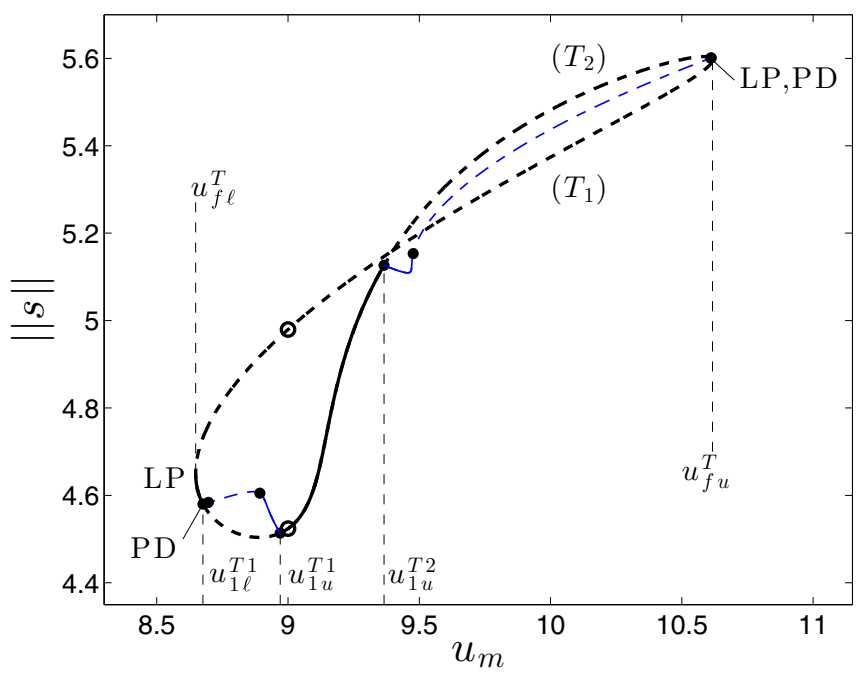

FIG. 12. Bifurcation diagram of the periodic dynamics $x_{p}$ [Eq. (17)] associated to the synchronized transport solutions $s_{T}$ with drift velocity $c= \pm 1$. The 1-periodic transport branches are delimited by two saddle nodes $u_{f \ell}^{T}=8.6483$ and $u_{f u}^{T}=10.6169$. Solution profiles at the locations indicated by circles are depicted in Fig. 11. Dots indicate period-doubling bifurcations, and fine blue lines are 2-periodic transport solutions. Unstable branches are indicated by a dashed line. The remaining parameters are as in Fig. $5(\gamma=10$, $a=0.65$ ).

$u_{1 u}^{T 1}=8.97$ and $u_{1 \ell}^{T 2}=9.37$, and the third range is very narrow in the vicinity of the upper fold $u_{f u}$ (not visible in Fig. 12). Both 2-periodic branches emerge from the period-doubling bifurcations (blue curves in Fig. 12). A similar route to chaos arises from the four period-doubling cascades. Namely, the cascade follows a geometric series and leads to a chaotic dynamics of $x_{p}$, which is bounded in to the comoving frame. Consequently, ranging from the stable periodic transport to the "bounded" strange attractor, the dynamics $x(t)$ is a stable transport with the mean velocity \pm 1 . We say that the velocity is locked or the transport is synchronized. Thus the dynamics can be chaotic and the transport velocity still locked. This synchronized transport is destroyed via a crisis. After the crisis, the attractor becomes unbounded in the comoving frame. As the extended attractor is symmetric in the laboratory frame, we believe that it appears through a merging crisis between attractors with opposite transport directions. The resulting dynamics presents alternations between transport to the left and to the right, and the transport velocity is no longer locked. We define the transport velocity $c(t)$ at the time $t$ as

$$
c(t)=\frac{x(t)}{t},
$$

and $c$ its limit if it exists. After the crisis, the particle trajectory visits alternately opposite ghost attractors with the velocity $c=+1$ or -1 . An example is given in Fig. 13. During a few tens of periods the dynamics is close to the transport solution with $c=1$ or -1 , and the change of direction needs only one or two time periods. The discrete dynamics at every discrete time $n$ is similar to a random walk [Fig. 13(a)]. The mean transport velocity remains close to zero as shown in Fig. 13(b). Therefore, the mean drift velocity drops from $|c|=1$ to 

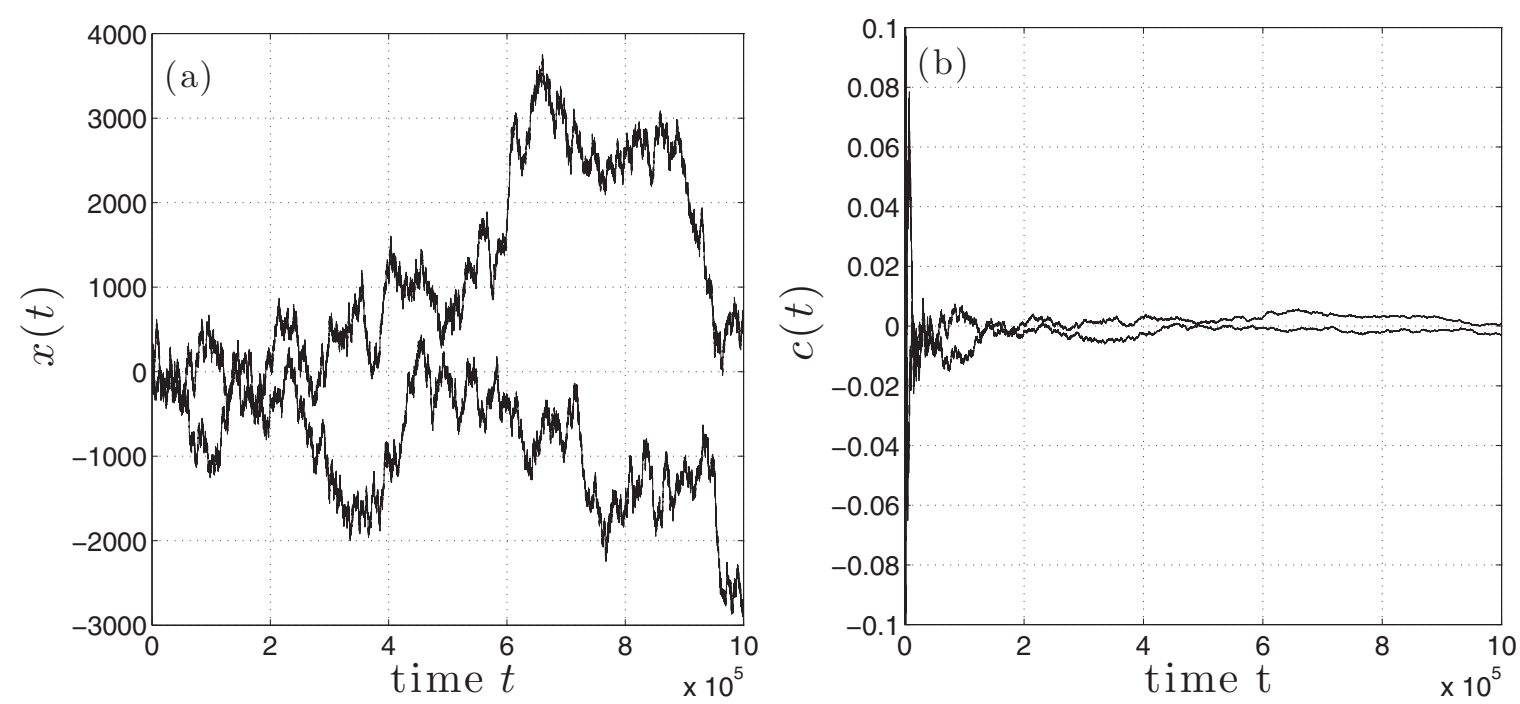

FIG. 13. (a) Particle dynamics in the unlocked velocity region of Fig. 12 for $u_{m}=8.8$ for two different initial conditions. The trajectories are reminiscent of a random walk. The remaining parameters are the same as in Fig. $12(\gamma=10, a=0.65)$. (b) Drift velocity $c(t)$ of the dynamics of panel (a). The velocity fluctuates around zero.

$c \simeq 0$. Figure 10 depicts the bifurcation scenario involving the periodic transport solutions.

We end the study of this branch by displaying the existence domain of the periodic solution in the parameter plane $\left(u_{m}, \gamma\right)$ (Fig. 14). Transport does not exist for $\gamma$ larger than 13 whatever the value of $u_{m}$. Thus, this transport exists only for moderate drag. For a decreasing drag, the existence domain is larger, yet the stability domain becomes thinner. This stability domain is very reminiscent of the existence domain of transport for an undamped ratchet problem in Ref. [24]. The path-following method allows us to find the instability domain and to distinguish the transitions as saddle node and period doubling. We further discuss, in Sec. V, the added value of such a bifurcation diagram.

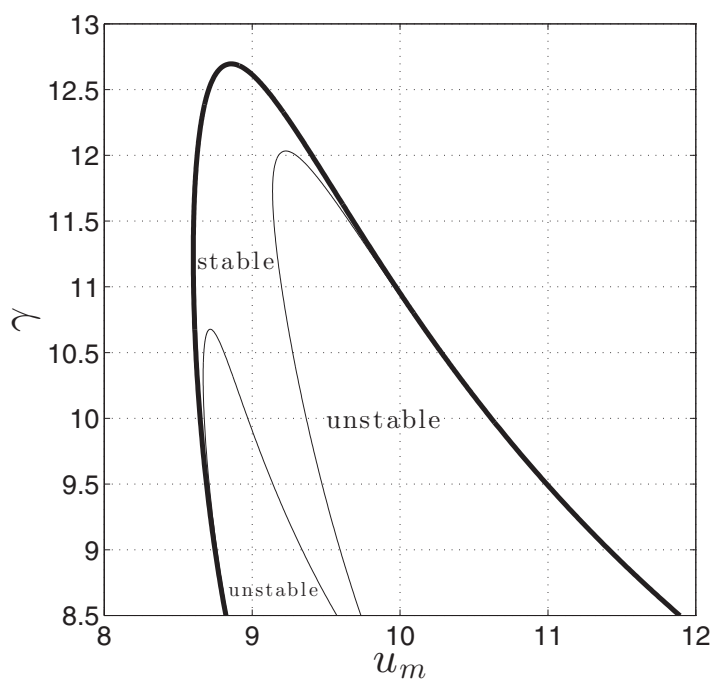

FIG. 14. Existence domain of the synchronized transport velocity with $c= \pm 1$ in the parameter plane $\left(u_{m}, \gamma\right)$. This domain is bounded by the fold bifurcation (bold line). The fine lines represent the transition between the stable and unstable solutions which is a period-doubling bifurcation.

\section{Role of the n-multiplying periodic solutions in the transport}

The scenario sketched in Fig. 10 shows that there is no direct bifurcation between periodic orbits and the transport solution. However, a necessary condition is the route to chaos via period doubling. In this subsection, we show that a quantitative property of the period-doubled solution can be a good indicator of the emergence of periodic transport.

In order to understand the possible link between the 2-periodic branch and the periodic transport solution, we represent in Fig. 15(a) the time evolution of the transport solution at its emergence $u_{f u}^{T}$ (Fig. 12) with 2-periodic solutions for the same parameters. At $u_{m}=u_{f u}^{T}$ among the three period-doubled branches in the bifurcation diagram Fig. 5(b), one chooses the $\left(B_{2}\right)$ branch because it has the largest difference between the two maxima as showed previously in Sec. III A 1 . This solution, noted $x_{2}^{(0)}$, is displayed by a circle in the bifurcation diagram [Fig. 5(b)], and it is located close to the turning point of $\left(B_{2}\right)$. Moreover, in Fig. 15(a) we also show the copies of $x_{2}^{(0)}$, noted $x_{2}^{(n)}$, and obtained by a space and a time period shift such as $x_{2}^{(n)}(t)=x_{2}^{(0)}(t+n)+n, n \in \mathbb{N}$. These solutions exist due to the discrete spatial and temporal shift symmetries. Figure 15 reveals that the trajectories of two consecutive orbits $x_{2}^{(n)}(t)$ and $x_{2}^{(n+1)}(t)$ are close for $t$ around $0.4+2 n, n \in \mathbb{Z}$. At this time, the velocity [the slope of $\left.x_{2}^{(n)}(t)\right]$ is maximal, and the particle position is about $\bar{x} \simeq 0.6$. Because the slopes of the two curves are almost equal, the two trajectories are close to each other in the phase space. If the period-two solution is not stable, the trajectory of the transport solution in the vicinity of $x_{2}^{(0)}$ may escape and switch to $x_{2}^{(1)}$ at $t$ about 0.4 as shown in Fig. 15: The synchronized transport solution $x_{T}$ is close to $x_{2}^{(0)}$ for $t$ between [0;0.4] and then moves progressively away from $x_{2}^{(0)}(t)$ and becomes closer to $x_{2}^{(1)}(t)$. Because of the spatiotemporal shift symmetry, this scenario occurs also in the same manner in the following pores, resulting in a synchronized transport with exactly $c=1$. Note that the opposite transport $\left(s_{T}^{-}, t+1 / 2\right)=\mathcal{S}\left(s_{T}^{+}, t\right)$ may 

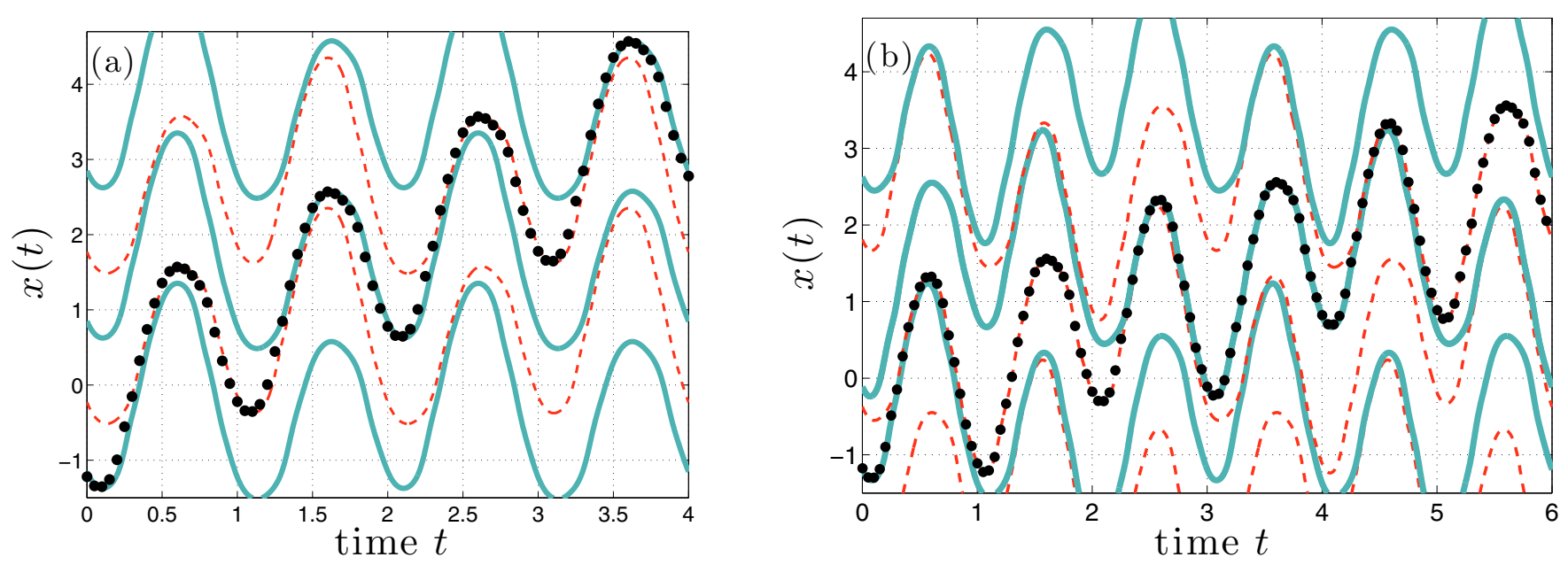

FIG. 15. (a) Time evolutions of (dots) the synchronized transport solution $(c=1)$ and (plain and dashed lines) of the period-two solution $x_{2}^{(0)}$ and its copies at $u_{m}=u_{f u}^{T}$ of the $\left(B_{2}\right)$ branch in Fig. 12. The plain lines correspond to the orbits $x_{2}^{(2 p)}(t)$ : copies of $x_{2}^{(0)}(t)$ shifted by $2 p$ spatial periods. The dashed lines correspond to the orbits $x_{2}^{(2 p+1)}(t)$ : copies of $x_{2}^{(0)}(t)$ spatially and temporal shifted by $2 p+1$ periods. During one period the transport solution is "guided" by the 2-periodic solution. Parameters $\gamma=10, a=0.65$, and $u_{m}=u_{f u}^{T}$. (b) Temporal evolution of the 3-periodic solutions and the $c=1 / 2$ transport solution. The plain and dashed black lines are period-three solutions translated in space and in time such as $x_{\text {dash }}(t)=x_{\text {plain }}(t+2)+1$. As in Fig. 15 the transport solution is guided during two periods by the 3-periodic solution. Parameters: $u_{m}=9, \gamma=13.153$, and $a=0.65$.

be explained by a similar mechanism of slip between periodic orbits using the symmetric period-doubled solutions $\mathcal{S}\left(s_{2}, t\right)$.

The role of the $\left(B_{2}\right)$ branch is enhanced by the fact that its existence domain $\left(\left[u_{f \ell} ; u_{f u}\right]\right.$ in Fig. 5) corresponds almost exactly to that of the transport branch $\left(\left[u_{f \ell}^{t} ; u_{f u}^{t}\right]\right.$ in Fig. 12). Indeed, contrary to $\left(B_{2}\right)$, the $\left(B_{1}\right)$ and $\left(B_{3}\right)$ branches present weak variations of the maximum during two temporal periods (see black dashed line in Fig. 6). As a result, the difference between the shifted trajectories constructed as previously remains about one, and transport is not expected.

Such a relationship between transport emergence and period-multiplying solution may be generalized for discrete velocity $c_{q}=1 / q, q \in \mathbb{Z}$.

This rational velocity means that the particle needs $q$ periods to advance exactly one period. For instance, a similar link can be found between the transport solution with $c=1 / 2$ and the three-periodic solution. In the time series [Fig. 15(b)] we represent a transport solution and an array of unstable 3-periodic solutions, which are shifted by one spatial period and two temporal periods, the solutions $x(t+2 p)+p, p \in \mathbb{Z}$. Again, we can point out the key role of periodic solutions, the period-three solution. Transport solutions remain in the vicinity of the periodic solution during two periods and then switch to another orbit when two consecutive orbits are very close [Fig. 15(b)]. Then the transport solution moves forward exactly one pore length during two temporal periods: $x_{T}(t+2 p)=x_{T}(t)+p$. Similarly to the case $|c|=1$, the transport solution emerges or ends at a tangent bifurcation. Moreover, this transport coexists with the period-three orbit and ceases when the periodic orbits are rather widely spaced.

\section{B. Effective transport for the asymmetric case $(d \neq 0)$}

If the parity symmetry of the flow is broken, i.e., $d \neq 0$, all asymmetric branches as $s_{a}$, period-doubled branches and the transport solutions found for the symmetric geometry should generically exist for $d$ small enough because of the continuity arguments. Thus by varying $d$, we examine the persistence of the transport solutions at moderate $\operatorname{drag}(\gamma \sim 10)$.

\section{Influence of the asymmetry $d$ on periodic solutions}

We begin by studying the influence of the asymmetry $d$ on the periodic branches as well as periodic transport solutions found in the symmetric case. The path following of these branches is performed by taking $d$ as bifurcation parameter and fixing $a, u_{m}$, and $\gamma$ to values which belong to the bifurcation diagram Fig. 5(b).

As shown in Appendix A, $s_{0}$ and $s_{m}$ still exist in the asymmetric case as long as $u_{m}$ is small. For parameters fixed in the neighborhood of $u_{m}=9, a=0.65$, and $\gamma=10$, the continuation of period-one solutions including $s_{a}$ shows that these branches exist for all values of $d$. Moreover, no bifurcation occurs when $d$ varies, and then they remain unstable. Regarding the asymmetric branches, they split into two distinct branches when $d \neq 0$. Thus, in the bifurcation diagram [Fig. 16] the three ( $B_{1,2}$ and 3 ) branches of the periodtwo solutions split into six branches when $d \neq 0$ and they remain unstable. One branch of $B_{1}$ and one of $B_{2}$ end at the saddle node $d=d_{f}^{p 2}>0.4$. We do not study in detail the consequence of this bifurcation since such a large asymmetry $d$ is not relevant for our model (see Sec. II B). Therefore, in the asymmetric case with a fixed $d<d_{f}^{p 2}$, the bifurcation diagram of the periodic solutions w.r.t. $u_{m}$ is similar to that in the symmetric case presented in Fig. 5.

In the same vein, each transport branch splits into two distinct branches $s_{T}^{+}$and $s_{T}^{-}$, the solutions of Eq. (17) with $c=+1$ and $c=-1$, respectively. The bifurcation diagram Fig. 17 shows that each transport solution, $s_{T}^{+}$and $s_{T}^{-}$, annihilates in a saddle-node bifurcation. The saddle nodes occur at different values (Fig. 17): $d_{f}^{-}=0.302$ (transport to 


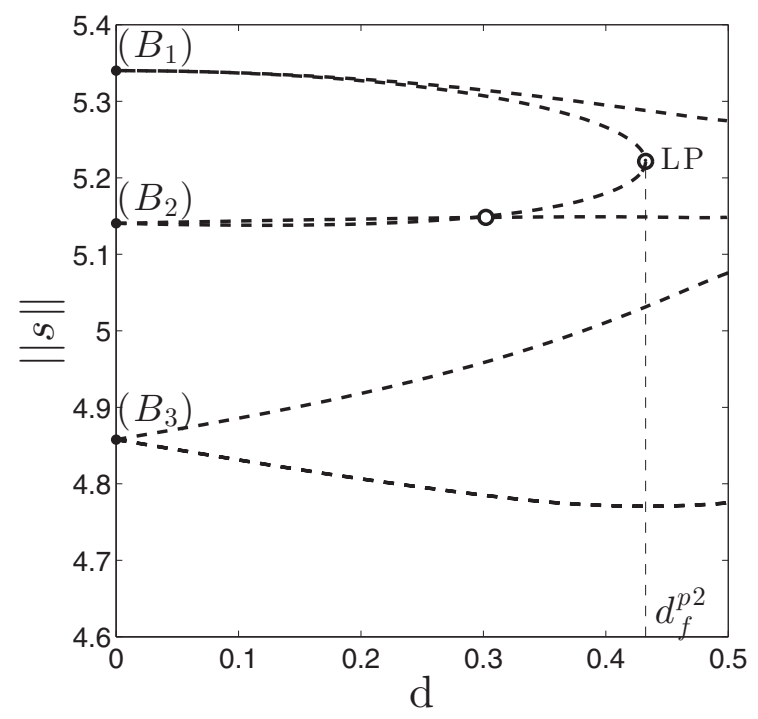

FIG. 16. Continuation of the 2-periodic branches starting from the $\left(B_{1}\right),\left(B_{2}\right)$, and $\left(B_{3}\right)$ branches of the bifurcation diagram Fig. 5(b) for $u_{m}=9$. Shown is the norm $\|s\|$ as a function of the asymmetry parameter $d$. Each of the three $s_{a}$ branches splits into two branches when $d \neq 0$. They are all unstable. Two branches meet at the saddle node noted $d_{f}^{p 2}$. Circles at $d=d_{f}^{-}$and $d_{f}^{+} \simeq d_{f}^{p 2}$ (fold bifurcations of Fig. 17) indicate specific solutions discussed in the beginning of Sec. III B. The remaining parameters are as in Fig. 5.

the left) and $d_{f}^{+}=0.433$ (transport to the right). No bifurcation takes place on the $s_{T}^{-}$branches. In contrast, the $s_{T}^{+}$branch presents routes to chaos and crises when $d$ varies (Fig. 17) via a scenario similar to the symmetric case (Fig. 10): the crisis marks the end of stable synchronized transport. Nevertheless,

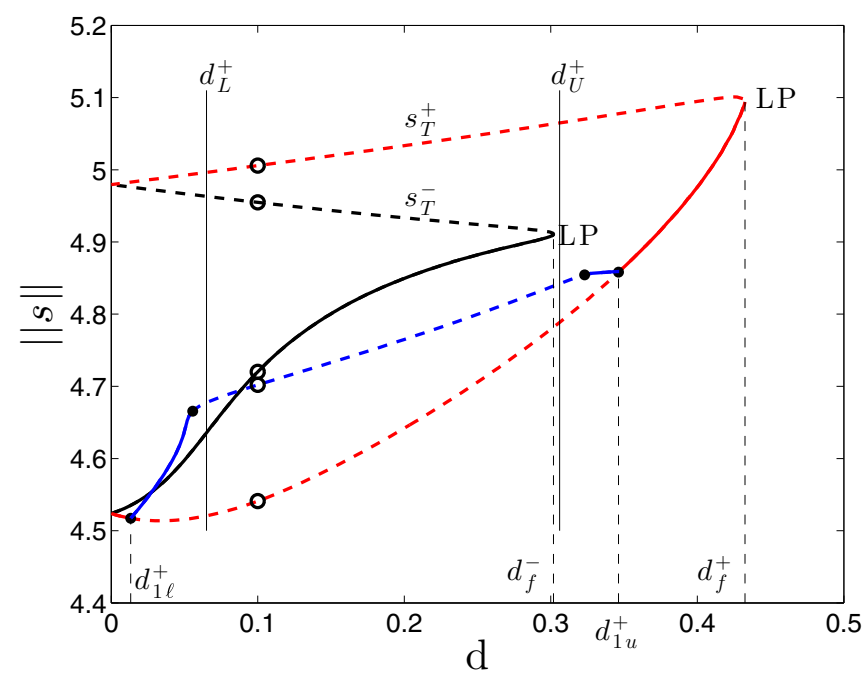

FIG. 17. Bifurcation diagram of the periodic transport solutions as a function of the asymmetry $d$. Starting solutions at $d=0$ correspond to circles at $u_{m}=9$ of the bifurcation diagram in Fig. 12. The red branch corresponds to $s_{T}^{+}$, the transport to the right, while the black branch corresponds to $s_{T}^{-}$, the transport to the left. The blue line displays the period-two transport solution of $s_{T}^{+}$. Solid (dashed) lines indicate stable (unstable) solutions, and dots designate period-doubling bifurcations. Circles at $d=0.1$ are the starting solutions of the bifurcation diagram (Fig. 18). the dynamics beyond the crisis can lead to a net drift because of the asymmetry. Let us describe the possible dynamics from the symmetric case $d=0$ till the fold $d_{f}^{+}$:

(1) From 0 to the crisis at $d_{L}^{+} \simeq 0.0657$, stable transports with $c=1$ and $c=-1$ coexist with different basins of attraction.

(2) The crisis marks the end of the strange attractor associated to the right transport. It is an attractor destruction crisis [55]. As mentioned in that reference, for $d>d_{L}^{+}$a transient chaotic dynamics occurs in the vicinity of $d_{L}^{+}$before it converges to the periodic left transport, the unique attractor till the fold bifurcation $d_{f}^{-}$. Then in the interval $\left[d_{L}^{+} ; d_{f}^{-}\right]$, a net transport with $c=-1$ is observed.

(3) In the narrow range $\left[d_{f}^{-}, d_{U}^{+}\right]$, only the periodic branches with $c=+1$ exist, but they are unstable. A chaotic dynamics takes place with a nonlocked velocity, which varies continuously from -1 to 1 . The dynamics involves switches between transports in opposite directions. Approaching $d_{U}^{+}$the phases of the transport to the right are longer. The transition crossing $d_{U}^{+}$by decreasing $d$ can be interpreted as a widening crisis: the chaotic attractor in the comoving frame $c=+1$ extends suddenly allowing transport episodes to the left. This situation is similar to the locked and unlocked velocity transition for the symmetric case. Nevertheless, a nonvanishing drift velocity seems to appear in the asymmetric case. Near the saddle node $d_{f}^{-}$, the dynamics will be detailed in the following subsection where the same kind of transition arises.

(4) For $d$ larger than $d_{U}^{+}$the transport to the right (chaotic or periodic) is an attractor of all the dynamics till $d_{f}^{+}$.

The continuous transition from $c=+1\left(d>d_{U}^{+}\right)$to $c=$ $-1\left(d=d_{f}^{-}\right)$by decreasing $d$, illustrates a current reversal scenario widely described in the literature on deterministic inertia ratchets, e.g., Refs. [22,24,27,57]. The reversal arises by way of a crisis $\left(d_{U}^{+}\right)$destroying the transport solution and followed by the birth of the opposite transport solution at a tangent bifurcation $\left(d_{f}^{-}\right)$. Note, however, that the occurrence of the saddle-node bifurcation of the left transport in the chaotic region of the right transport is only a coincidence. Indeed, there are other possible scenarios as we show in the next bifurcation diagram (Fig. 18).

\section{Current reversal}

In this section, we focus on the current reversal due to the variation of the drag $\gamma$ and not due to a change in the pore geometry, which can be achieved by modifying the pumping frequency. Then the parameter $d$ is fixed, and the drag $\gamma$ is considered as the bifurcation parameter. We start from the transport solution found at $d=0.1$ in Fig. 17. Each branch, $s_{T}^{+}$and $s_{T}^{-}$, presents a scenario identical to that displayed in Fig. 10. They emerge or end at a lower fold tangent bifurcation noted $\gamma_{f \ell}^{ \pm}$or at an upper fold tangent bifurcation noted $\gamma_{f u}^{ \pm}$ where \pm designates the transport direction [Fig. 18]. In its existence domain, crises involving synchronized transports occur; they are noted $\gamma_{L}^{ \pm}$and $\gamma_{U}^{ \pm}$in Fig. 18. Owing to the fact that the existence domains of the opposite transport solutions and the bifurcation scenarios (Fig. 10) do not match, there are intervals of $\gamma$ where there is only one stable transport solution. Four bands are displayed in Fig. 18 corresponding to 


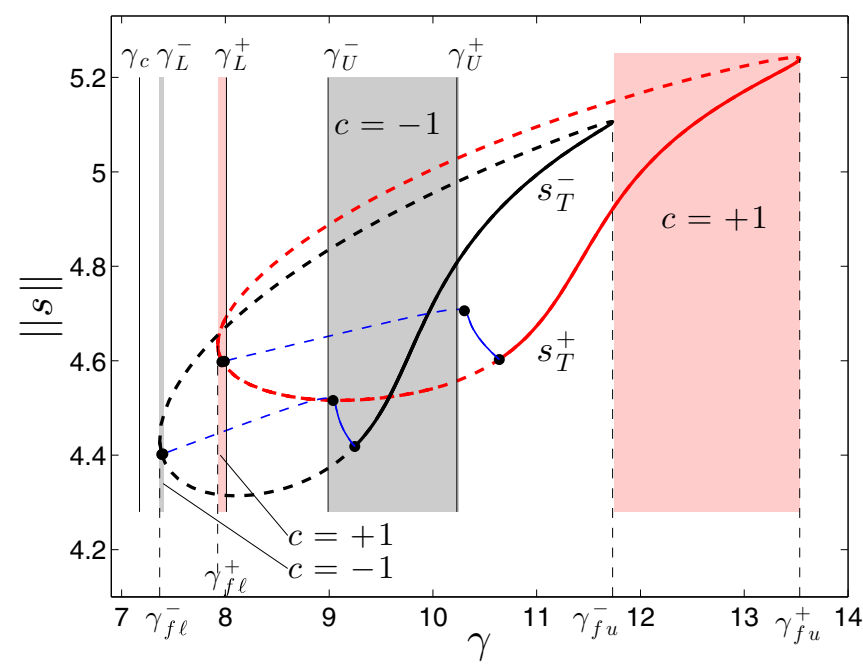

FIG. 18. Bifurcation diagram of the periodic transport solutions to the right $\left(s_{T}^{+}\right.$black line) and to the left $\left(s_{T}^{-}\right.$red line). Shown is the norm $\|s\|$ as a function of the drag coefficient $\gamma$ for the asymmetric case $d=0.1$. At $\gamma=10$ the solutions are the circles displayed in Fig. 17. The color, stability, and bifurcation codes as in Fig. 17. Both transport solutions have a similar bifurcation scenario as for the symmetric case (Fig. 10), especially the emergence of the branches at saddle nodes $\left(\gamma_{f \ell}^{-}, \gamma_{f \ell}^{+}, \gamma_{f u}^{-}, \gamma_{f u}^{+}\right)$and windows of unlocked transport velocity via crisis. The crises corresponding to the onset of unlocked velocity are indicated by the vertical lines $\gamma_{U}^{+}, \gamma_{U}^{-}, \gamma_{L}^{+}$, and $\gamma_{L}^{-}$. The red and gray areas correspond to ranges of stable synchronized transport with $c=1$ and $c=-1$, respectively. The gray area near $\gamma_{f \ell}$ is inflated in order to be visible. The current reversal occurs between these four regions. The vertical line at $\gamma=\gamma_{c}$ is the onset of unbounded dynamics. Parameters: $a=0.65, u_{m}=9, d=0.1$.

these stable transports with $c=1$ or -1 . It follows that three scenarios of current reversal exist. They are by decreasing $\gamma$ :

(1) In the range $\left[\gamma_{f u}^{-} ; \gamma_{f u}^{+}\right]$, the dynamics is attracted by the unique stable transport to the right, while in the interval $\left[\gamma_{U}^{-} ; \gamma_{U}^{+}\right]$, the transport $c=-1$ is the unique attractor. Thus,

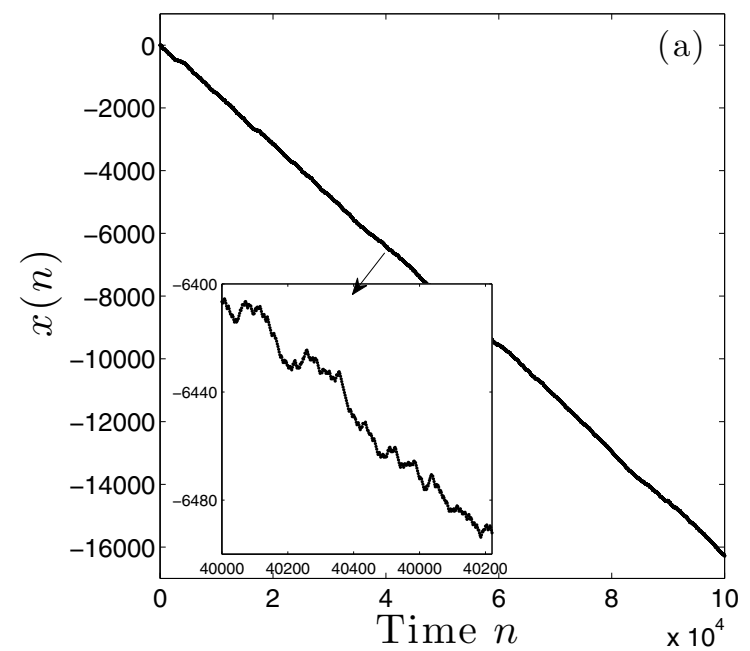

the current reversal occurs in the bistability range $\left[\gamma_{U}^{+} ; \gamma_{f u}^{-}\right]$. Depending on the initial conditions the dynamics is attracted by one of these two stable solutions. If one takes the mean velocity over the initial conditions as in Ref. [22], then the mean drift velocity varies continuously from 1 to -1 when $\gamma$ decreases from $\gamma_{f u}^{+}$to $\gamma_{R}^{+}$. Such a transport reversal was already interpreted as a consequence of bistability in Refs. [23,24].

(2) The second current reversal transition takes place between the two crises $\gamma_{U}^{-}$and $\gamma_{L}^{+}$. The crisis at $\gamma_{U}^{-}$corresponds to a widening crisis as is the case for the crisis at $d_{U}^{+}$in bifurcation diagram Fig. 17. Beyond and close to the crisis $\left(\gamma<\gamma_{U}^{-}\right)$, the dynamics is intermittent: The particle trajectory spends long stretches near the synchronized left transport, intermingled with bursts in the new region of the widened attractor. The dynamics during the burst reveals a slow drift to the right. Thus the absolute drift velocity decreases. Away from this crisis, the drift to the right is longer and the time integration provides trajectories alternately close to that of the transport solutions [Fig. 19(a)]. Moreover, the switch between the directions lasts less than one period; i.e., one does not observe the events of bounded motions. Because of the asymmetry, the duration in the vicinity of each transport direction is not necessarily equal and the simulation shows a convergence to a limit velocity $c$ [Fig. 19(b)]. Thus, the drift velocity varies continuously from -1 to 1 when $\gamma_{U}^{-}$varies to $\gamma_{L}^{+}$where a reverse widening crisis occurs.

(3) For $\gamma \leqslant \gamma_{f \ell}^{+}=7.927$, the right transport with $c=1$ ceases to exist. Time integration with parameters close to the threshold shows epochs of near periodic transport with $c=+1$ interspersed by a chaotic dynamics with a small negative drift. The duration of chaotic events remains long even for $\gamma$ very close to the onset. For instance, for a relative distance from the onset $\gamma_{f \ell}^{+}$about $10^{-9}$ the duration of chaotic dynamics events is longer than for the regular, nearly periodic, transport [Fig. 20(a)]. The resulting dynamics is a slow drift about $c \simeq$ 0.1 [see Fig. 20(b)]. Therefore, crossing the critical value, the mean value jumps from +1 to a small value. On decreasing

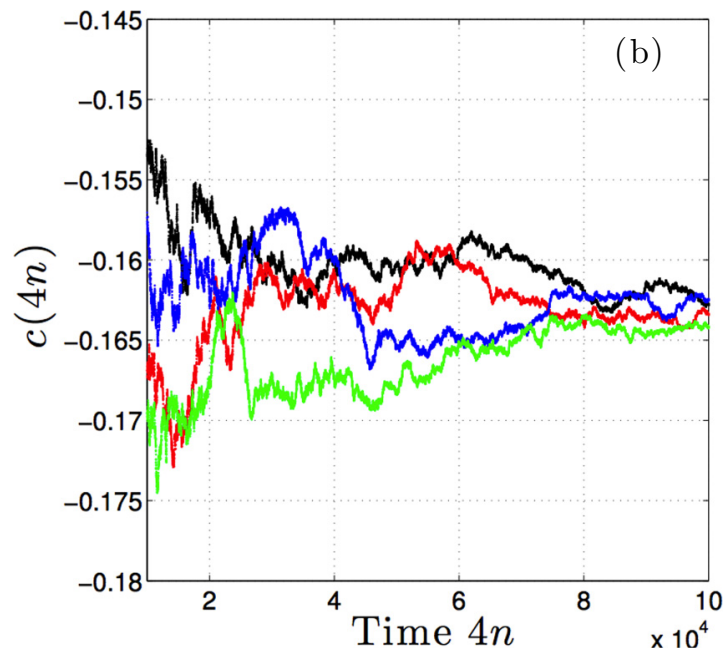

FIG. 19. (a) Stroboscopic particle position $x(n)$ at discrete times for $\gamma=8.8$ with the parameters of the bifurcation diagram in Fig. 18 $\left(u_{m}=9, a=0.65, d=0.1\right)$. The dynamics displays a net drift to the left. The inset shows that the drift results from a competition between transport in opposite directions. (b) Time evolution of the transport velocity $c(t)$ for four different initial values. The drift velocity fluctuates around -0.163 . 

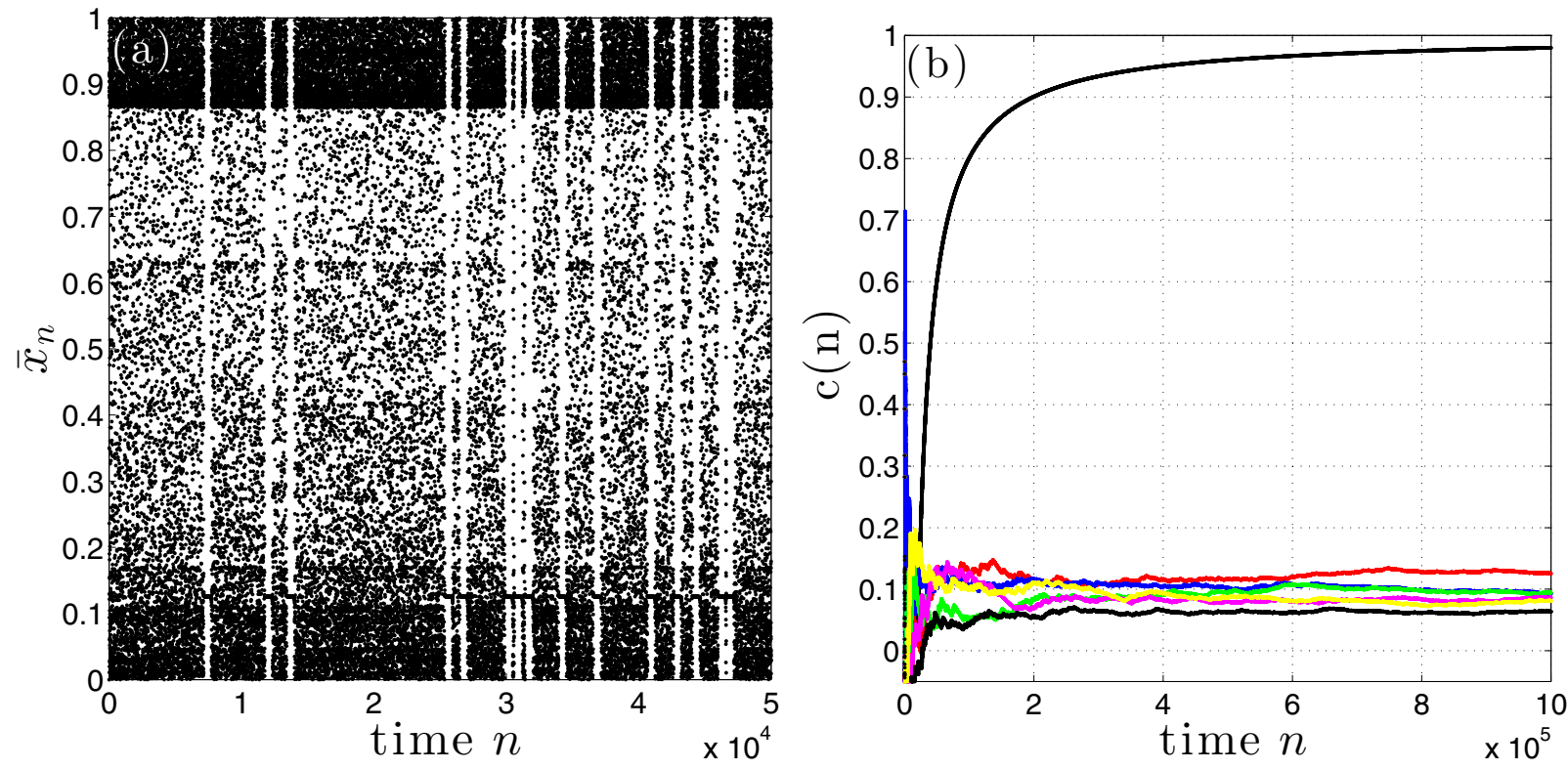

FIG. 20. (a) Relative particle position $\bar{x}_{n}=\bar{x}(n)$ for parameters near the onset of periodic transport $\gamma_{f \ell}^{+}$(Fig. 18): $\gamma=7.9261516, u_{m}=9$, $a=0.65$, and $d=0.1$. The horizontal lines at $x \sim 0.13$ in the blank spaces indicate epochs of near periodic transport. (b) Drift velocity $c(n)$ of dynamics obtained for $\gamma$ ranging from 7.92615100 to $7.92615167 \simeq \gamma_{f \ell}^{+}$[other parameters being the same as in panel (a)]. The black curve converging to $c=1$ corresponds to the dynamics at the onset $\gamma_{f \ell}^{+}$. The other curves (in color) range from 7.92615150 to 7.92615166 and fluctuate around 0.1.

the bifurcation parameter further, the transport events to the right vanish. Only transport events to the left remain, and they alternate with bound dynamics remains (Fig. 21). The current reversal finishes at $\gamma_{L}^{-}$onset of a widening crisis as it occurs for $\gamma_{L}^{+}$.

To conclude, the asymmetry shifts the existence and stability domains of the opposite transport solutions which coexist in the symmetric case. Therefore the transport reversal is an associated phenomenon to synchronized transport provided with a small asymmetry. That is why this phenomenon is reported in many papers involving synchronized transport

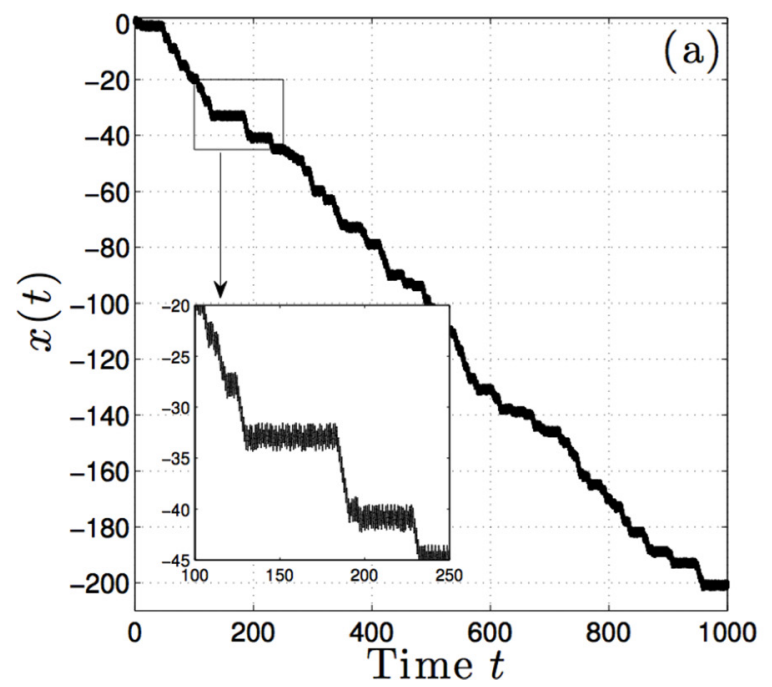

solutions. Note that it is difficult to find the ends of the current reversals in the narrow ranges $\left[\gamma_{f \ell}^{-}, \gamma_{L}^{-}\right]$and $\left[\gamma_{f \ell}^{+}, \gamma_{L}^{+}\right]$without knowledge of the periodic solution branches. The bifurcation diagram of the periodic transport branch is therefore a powerful tool to detect the current reversal scenarios.

\section{Transitions between transport and bounded dynamics}

As for the symmetric case, the scenario from periodic transport to periodic orbit involves two transitions [Fig. 10]: (1) saddle node from periodic transport to chaotic transport

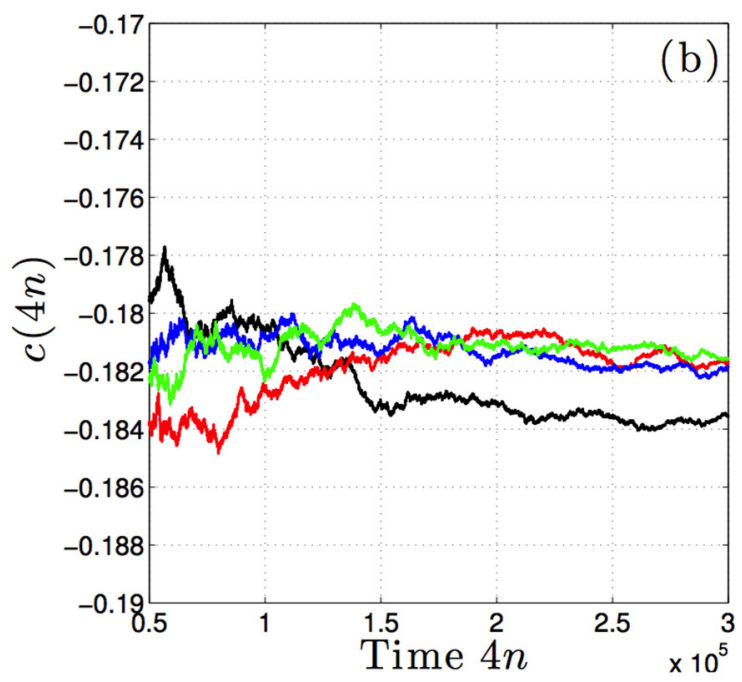

FIG. 21. (a) Time evolution of the particle position for $\gamma=7.5$ in the region of unlocked velocity of the bifurcation diagram Fig. 18 ( $u_{m}=9, a=0.65, d=0.1$ ). We observe long periods of oscillations in one pore followed by transport episodes leading to a slow drift to the left. (b) Time evolution of the transport velocity $c(t)$ for four different initial values. 

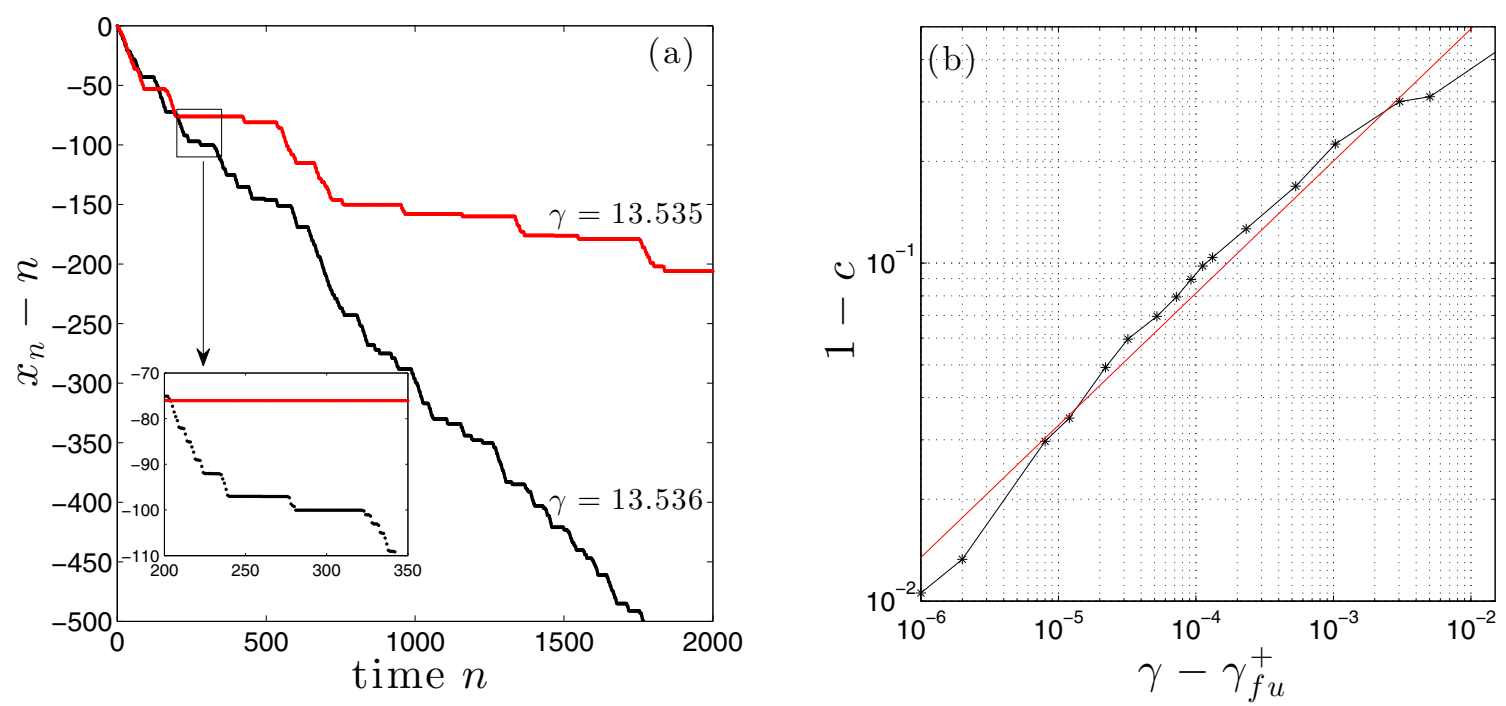

FIG. 22. Stroboscopic particle position at discrete times $n$ in the comoving frame $(c=+1)$ for two different values of $\gamma$ close to the saddle node $\gamma_{f u}^{+}$(see Fig. 18). The dynamics displays an irregular staircase. Statistically the plateau length increases and diverges when $\gamma$ approaches the tangent bifurcation. Remaining parameters are as in Fig. 18.

and (2) a crisis from unbounded chaotic dynamics to bounded chaotic dynamics. Before we detail these transitions, note that we found a similar relationship to that described in Sec. III A 4 between 2-periodic solutions and the periodic transport. Indeed, the plot (not shown) of the time evolution of the transport solutions at each onset $d_{f}^{-}$and $d_{f}^{+}$together with the period-two solutions (dots in Fig. 16) displays the same qualitative behavior as in Fig. 15: the transport trajectory is close to a period-two orbit during one temporal period and switches to the next spatially shifted orbit during the following temporal period. Moreover it is noteworthy that the domain of existence of the transport solution to the right almost matches that of the period-two branch: $d_{f}^{+} \simeq d_{f}^{p 2}$. Therefore, the fact that shifted orbits are close to each other still appears in the asymmetric case as an indicator of the possible emergence of periodic transport.

(1) We study, first, the transition starting from the critical value $\gamma_{f u}^{+}$corresponding to the upper limit of the periodic transport domain. The time integration of the solution in the comoving frame $x(t)-t$ for $\gamma>\gamma_{f u}^{+}$, shows long plateaux corresponding to a transport with velocities very close to plus one [Fig. 22(a)]. These epochs of transport are interrupted by short irregular dynamics. The resulting discrete dynamics $x_{n}$ resembles a descending staircase with irregular steps [Fig. 22(a)]. The fact that the staircase is always descending implies that the transport $c=1$ is the maximum velocity. Contrary to the symmetric case, there is a net transport to the right, and the transport events to the left are short. The lengths of the plateaux become statistically longer as one approaches the fold bifurcation. It is possible to roughly define a mean velocity [Fig. 22(b)]. By "roughly," we mean that $c(t)$ fluctuates around a mean value. Increasing further $\gamma$ (away from the onset), the transport events are shorter, thus the mean velocity decreases continuously. Figure 22(b) shows that the relative velocity $1-c$ follows a power law with an exponent 0.39 . Increasing $\gamma$ further, the plateaux are shorter and the drift velocity decreases. However, we are not able to detect the transition from intermittent transport to bounded dynamics because of the presence of a synchronized transport with $c=+1 / 3$ which attracts all the dynamics for $\gamma$ about 14.2.

The intermittent behavior near the lower boundary $\gamma_{f \ell}^{-}$ of the existence domain of periodic transport resembles the transition at $\gamma_{f \ell}^{+}$discussed in the current reversal scenarios. More precisely, for a relative variation of $\gamma$ from $\gamma_{f \ell}^{-}$inferior to $10^{-7}$, we obtain a similar figure to Fig. 20: the plateaux of regular dynamics are about 300 periods, while the chaotic dynamics is about $3 \times 10^{4}$. Closer to the onset, the plateau length increases, but the duration of the chaotic dynamics remains larger. When the stable solution exists, i.e., $\gamma>\gamma_{-}^{f \ell}$, a long chaotic transition arises before it converges to the stable left transport. These results suggest the presence of a nonattracting chaotic invariant set, i.e., a repeller, in the neighborhood of the fold bifurcation. Thus, because of the possible attracting manifold of the repeller, the dynamics explore the neighborhood of this repeller before approaching the ghost periodic transport solution and so on. From the perspective of transport, as during the long chaotic events the drift is small, this results in a slow transport to the left still close to the onset. Therefore, on passing the onset, the velocity varies dramatically from $c=-1$ to a small negative value. This behavior is analogous as it happens at the $\gamma_{f \ell}^{+}$transition. (2) Decreasing $\gamma$ further, the transition to the bounded dynamics is found at $\gamma \simeq 7.172$. The simulation close to this point displays a vanishing drift (Fig. 23). As in the symmetric case (see Fig. 8) long epochs of bound dynamics are interspersed by short drifts due to a crisis between consecutive attractors. The bounded dynamics is like a quasiperiodic motion with period two as suggests the double plateau in Fig. 23(b). This bounded attractor is obviously not symmetric and the crisis becomes a widening crisis instead of a merging crisis as in Sec. III A 2. Hence, the dynamics of the widened 

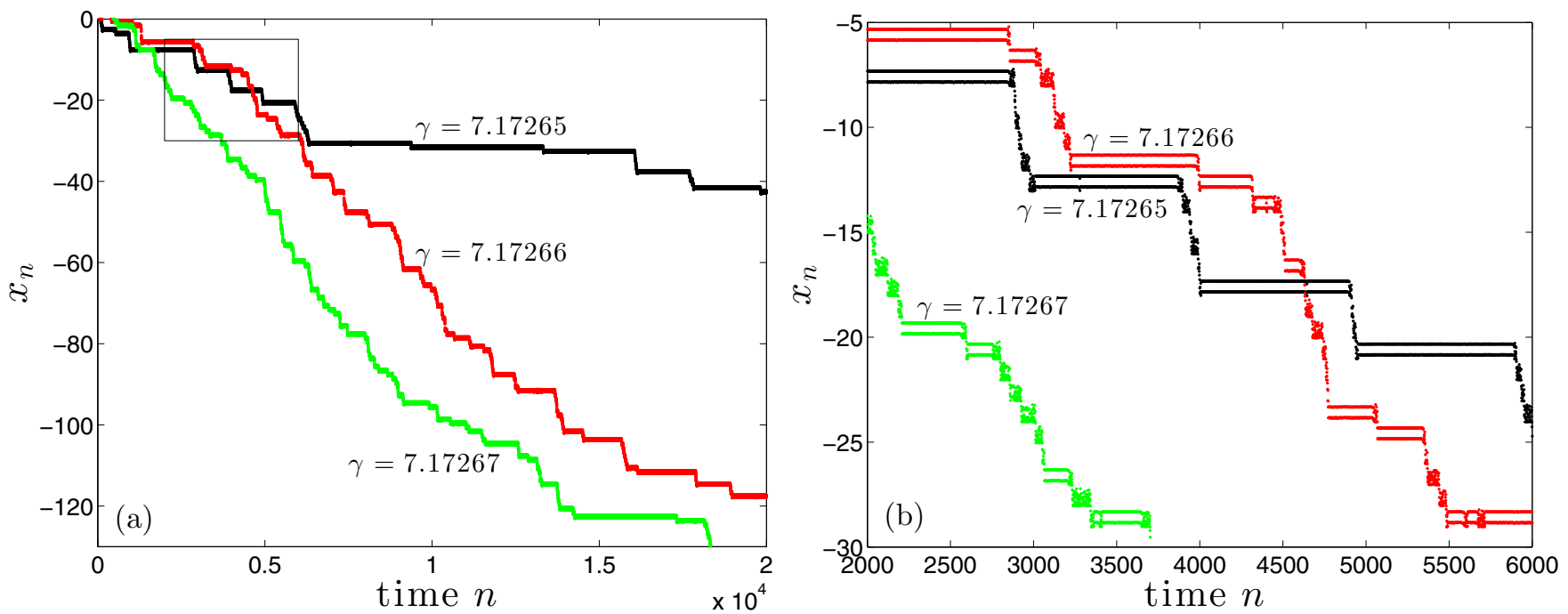

FIG. 23. (a) Particle position at discrete times $n$ close to the threshold of unbounded dynamics at $\gamma_{c} \simeq 7.1726$ (Fig. 18). (b) Magnification of the rectangle in panel (a). The plateaux are nearly 2-periodic, while the short transport events display a chaotic behavior. The drift velocity vanishes rapidly on approaching the threshold. Remaining parameters as in Fig. 18.

attractor causes drift events only to the left, leading to a net transport.

In conclusion, the emergence of the transport is due to a crisis of the consecutive shifted attractors on the one hand, and on the other hand, the periodic transport appears at a saddle node and corresponds to the largest drift velocity.

\section{TRANSPORT VIA FORCED SYMMETRY-BREAKING}

The asymmetric case provides a transport mechanism for particles with large drag. As shown in Sec. III A 1, when the drag is about 100 the dynamics is attracted by a 1-periodic solution in a symmetric geometry. In contrast, by varying the asymmetry $d$, we find that this solution may disappear and transport emerges even if the drag $\gamma$ is large. Then, contrary to the previous section the transport is induced by the asymmetry. We detail below the kind of transport found.

The bifurcation diagram (Fig. 24) of the period-one solutions in the symmetric case is expanded by using the velocity contrast $a$ [see Eq. (10)] as a bifurcation parameter and fixing the drag and the characteristic velocity: $\gamma=100$ and $u_{m}=9$. The period-one solutions $s_{0}$ and $s_{m}$ exchange their stability via two pitchfork bifurcations and are connected by the asymmetric $s_{a}$ branch (Fig. 24) as in Fig. 4. The $s_{a}$ branch exists in the ranges surrounding $a=0.65\left(\left[a_{m} ; a_{M}\right]\right.$ inset of Fig. 24) and $a=0.9$. Now, let us study the existence of these solutions for an asymmetric velocity profile, i.e., when $d$ varies. It is found that if $a$ is smaller than the critical value $a_{c}=0.507$, then both branches $s_{0}$ and $s_{m}$ still exist for all values of $d$ and their stability does not change. When $a$ is superior to $a_{c}$, the two branches annihilate in a fold bifurcation at $d_{f}<1 / 2$ (Fig. 25). Note that a saddle-node bifurcation between $s_{0}$ and $s_{m}$ involving the coalescence of their mean positions is not possible within the symmetric case since they have distinct mean positions. The time integration of Eq. (4) with $d>d_{f}$ and close to $d_{f}$ displays a slow drift to the left. The drift is intermittent: the particle remains during many periods in the vicinity of the vanished periodic solution and then drifts to the next pore. The stroboscopic time evolution of the particle position at every period displays a regular descending staircase for different values of $d$; the plateaux correspond to oscillations close to the threshold (Fig. 26). The plateaux become longer when $d$ approaches $d_{f}$. The computation of the drift velocity $c$ as a function of $d-d_{f}$ indicates a power law dependence: $c \sim\left(d-d_{f}\right)^{1 / 2}$ (Fig. 27). This power law is consistent with the type-I intermittent bifurcation [58,59]. To corroborate that, the return map $\left(\bar{x}_{n}, \bar{x}_{n+1}\right)$ of the discrete positions $x_{n}$ of the particle position at every period $t=n$ is plotted in Fig. 27. The return map displays a smooth curve tangent to the bisectrix as is observed near the threshold of type-I intermittency for the one-dimensional problem $x_{n+1}=f\left(x_{n}\right)$. A priori, our problem is two-dimensional and the iteration map should depend on

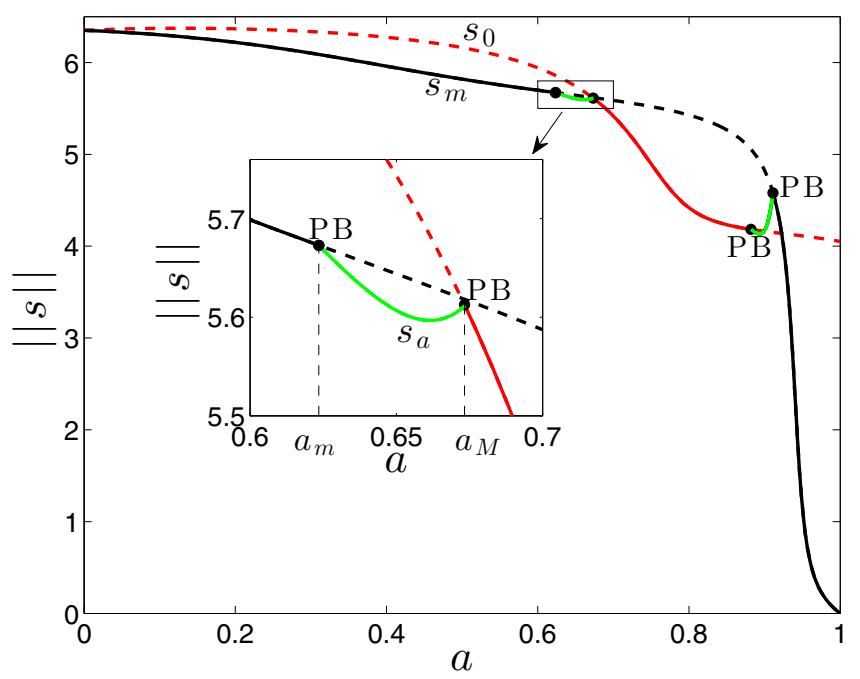

FIG. 24. Bifurcation diagram showing the norm $\|s\|$ of the one-periodic orbits $s_{0}, s_{m}$, and $s_{a}$ for the symmetric velocity profile as a function of the velocity contrast $a$. The pitchfork bifurcations are indicated by dotted lines. Plain [dashed] lines indicate stable (unstable) solutions. Parameters $\gamma=100, u_{m}=9$, and $d=0$. 

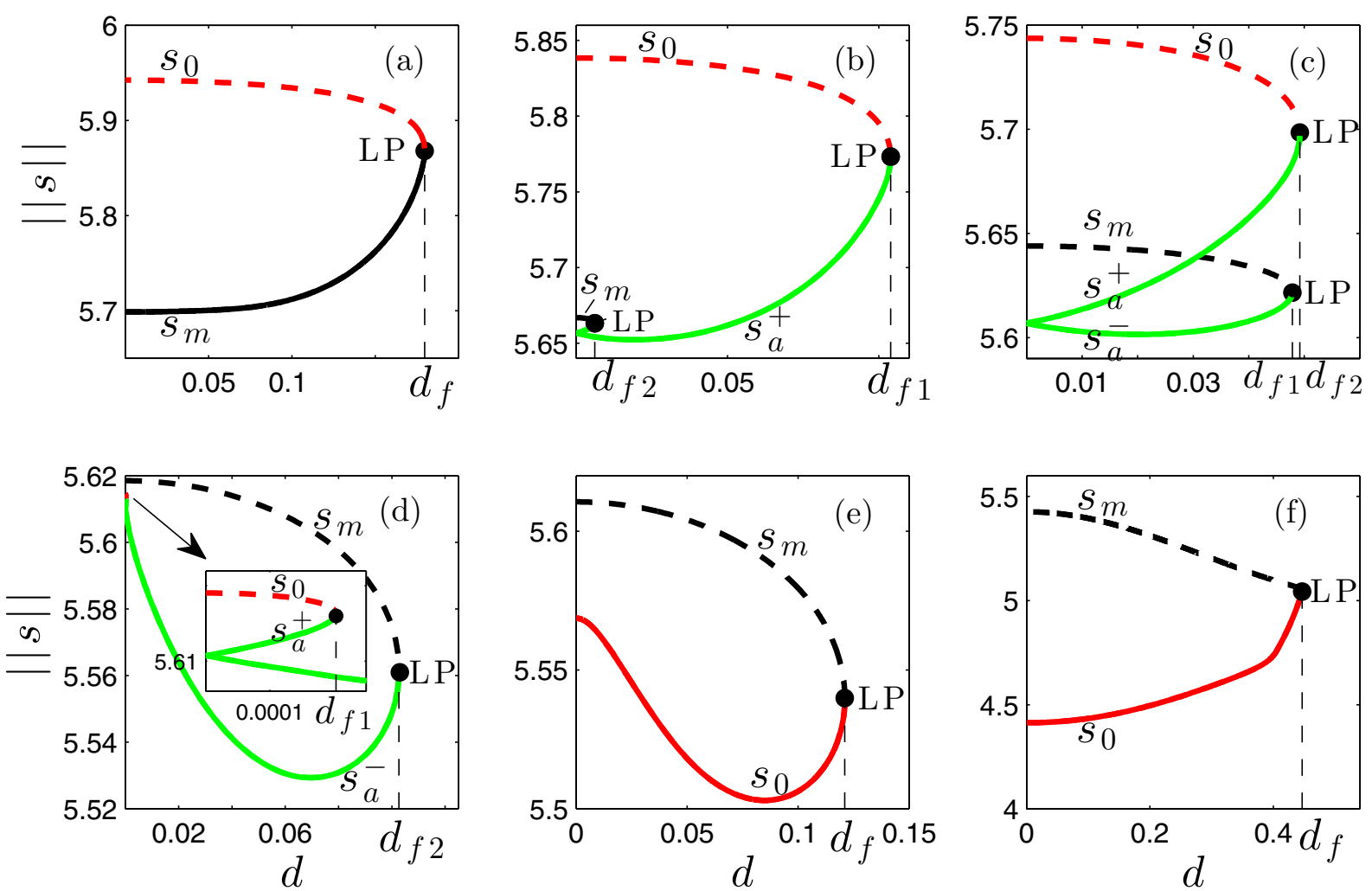

FIG. 25. Continuation of the branches $s_{0}, s_{m}$, and $s_{a}$ starting from the symmetric case (Fig. 24) by varying the $d$ parameter and for different values of $a$ : (a) $a=0.6$, (b) $a=0.628$, (c) $a=0.65$, (d) $a=0.673$, (e) $a=0.68$, and (f) $a=0.8$. All the branches end at a saddle-node bifurcation noted $d_{f}$. In (b)-(d), the $s_{a}$ branch splits into two branches $s_{a}^{+}$and $s_{a}^{-}$involving the existence of two saddle nodes noted $d_{f 1}$ and $d_{f 2}$. Color code as in Fig. 24. Remaining parameters are $\gamma=100, u_{m}=9$.
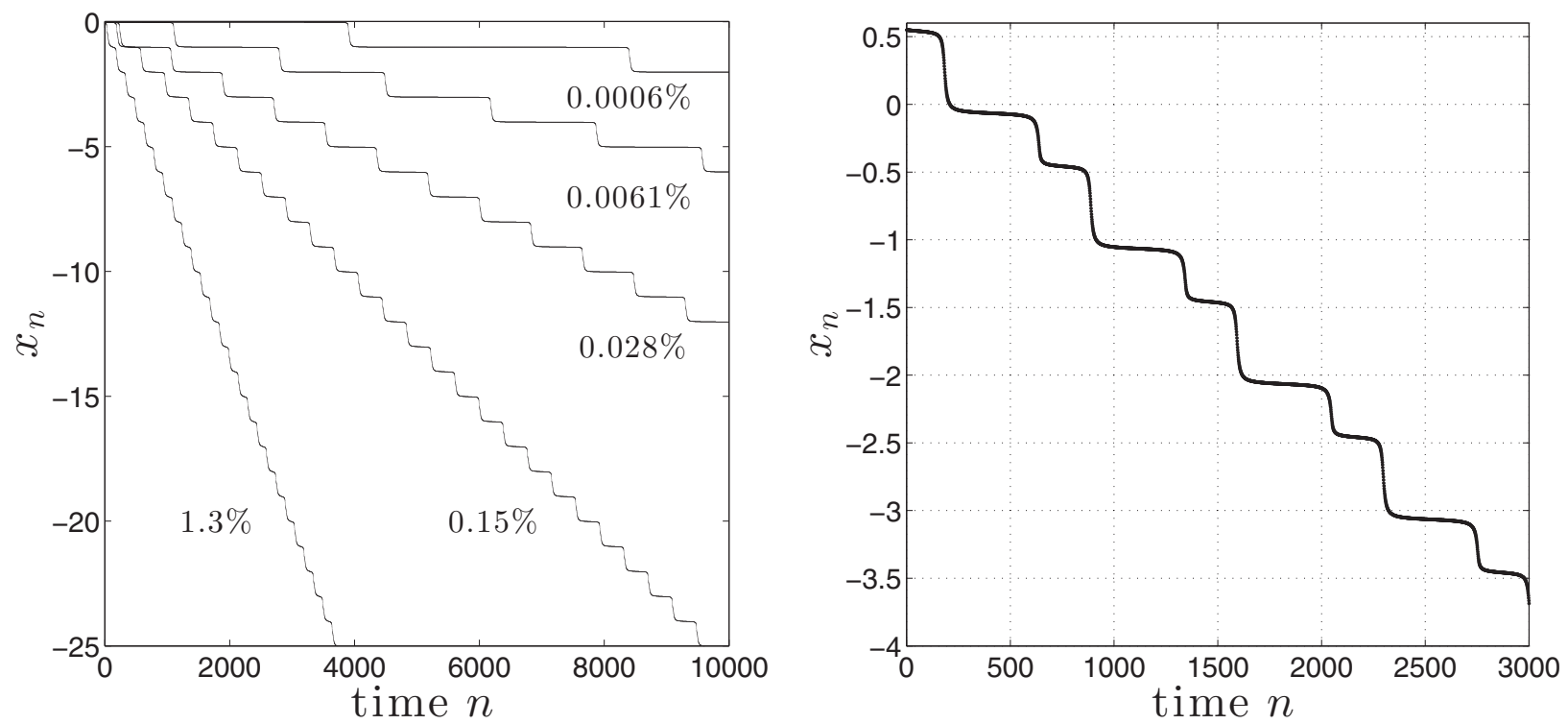

FIG. 26. Stroboscopic position $x_{n}$ at discrete times of the dynamics for different parameters. Long plateaux of nearly 1-periodic motions are regularly interrupted by a drift to the next pore. (Left) $a=0.6$ and $d$ takes different values superior to the threshold $d_{f}$ of Fig. 25 . The percentage of the relative $d$ variation $\left(d-d_{f}\right) / d_{f}$, is indicated below each plot. (Right) $a=0.65$ and $d=0.0494$ superior to the onset $d_{f 1}$ in Fig. 25(c). Each plateau is close to a fixed point at one of the two saddle nodes $d_{f 1}$ and $d_{f 2}$. Common parameters: $\gamma=100, u_{m}=9$. 

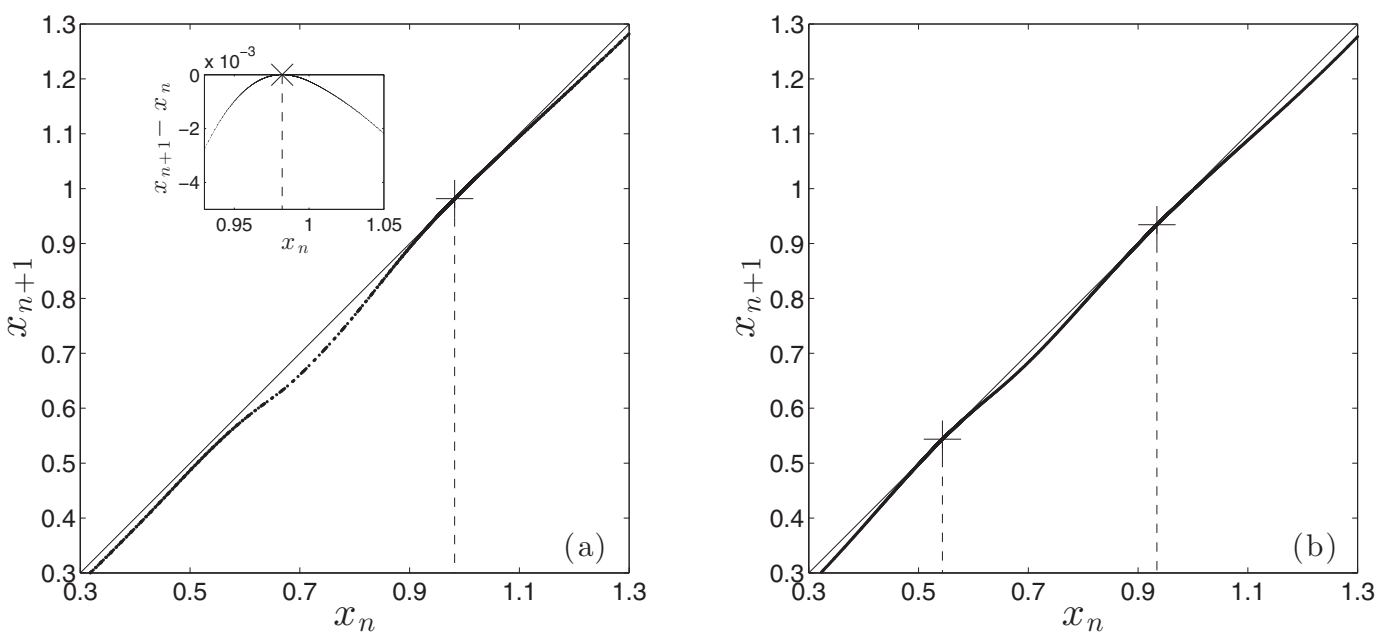

FIG. 27. Return map of the particle position $x_{n}$ at discrete times $n$ of the dynamics close to the intermittent bifurcation $d_{f}$ for (a) $a=0.6$ and (b) $a=0.65$. The " + " symbol indicates the fixed point at $d_{f}$. For panel (b) there are two fixed points according to Fig. 25(c). The inset of panel (a) shows that the curve passes very close to this point, but it does not cross the bisectrix. Remaining parameters as in Fig. 26.

the velocity $v_{n}$ too. But the one-dimensional aspect of the dynamics is a consequence of the large drag. According to Eq. (4):

$$
x_{n+1}-x_{n}=\int_{0}^{1} u_{0}[x(t)] \sin 2 \pi t d t-\frac{1}{\gamma}\left(v_{n+1}-v_{n}\right)
$$

Because $1 / \gamma$ is small and the particle velocity $v$ is bounded, the last term of the right-hand side is negligible:

$$
x_{n+1}-x_{n} \simeq \int_{0}^{1} u_{0}[x(t)] \sin 2 \pi t d t=g\left(x_{n}\right) .
$$

The regular particle drift contrasts with the usual intermittent dynamics as in the previous Sec. III B (Figs. 22 and 23). It could be related to periodic shadowing orbits [60]; i.e., the trajectory stays "close" to an unstable periodic transport of large period. But to find such a periodic solution is a very difficult task [61]. Therefore, to explain the drift regularity, we propose to study the approximated one-dimensional discrete dynamical system (20) where (1) $g$ is 1-periodic, small, and strictly negative and (2) $\left|g^{\prime}\right|$ is not large compared to one. Indeed, the spatial periodicity implies the periodicity of $g$ [Eq. (20)] and the quasiadvective motion for large drag implies that the drift $x_{n+1}-x_{n}=g\left(x_{n}\right)$ is small. Furthermore, due to the fact that the flow velocity gradient $\frac{\partial u_{0}}{\partial x}$ is not large then, according to Eq. (20), the derivate $g^{\prime}$ acquires this property too. Finally, the assumption $g<0$ is related to the fact that there is no periodic solution beyond the fold bifurcation and thus $g$ cannot change its sign. For this particular example, the drift is negative then $g$ is strictly negative.

Under these assumptions, we prove that a particle needs $N$ or $N-1$ temporal periods to pass through one pore, where $N$ does not depend on the initial condition (see Appendix B). This explains the regularity of the staircase in Fig. 26 and the attraction property of the whole dynamics since it is true for any initial condition. The simulation fairly corroborates this result. For instance, let us consider the case $a=0.6$ where $d_{f}$ is equal to 0.1797384 . For $d=0.18000$, the number of periods is 378 or 379 while for $d=0.17975$ it is 1692 or 1693 . However, very close to the critical point this rule is no longer valid:
When $d=d_{f}+10^{-7}$, the number of temporal periods varies between $N-250$ and $N+250$ with $N \simeq 18000$. This can be explained by the two-dimensional aspect of the dynamics. In fact, if $\left|d-d_{f}\right|$ is small, the step length vanishes, and then the dropped term $\left(v_{n+1}-v_{n}\right) / \gamma$ in Eq. (20) is no longer negligible. Nevertheless, the mean value of $N$ is large compared to its fluctuations, and the drift therefore still appears regular.

Let us briefly describe the other transport transitions for other values of the contrast $a$, in particular when the $s_{a}$ branch is present [Figs. 25(b)-25(d)]. Starting from $d=0$, this branch splits into two branches $s_{a}^{+}$and $s_{a}^{-}$, which connect the branches $s_{0}$ and $s_{m}$ at two turning points $d_{f 1}$ and $d_{f 2}$, respectively. For $a$ close to $a_{m}$, the critical value $d_{f_{2}}$ is close to zero [Fig. 25(b)], and it increases with $a$ while $d_{f 1}$ decreases until it vanishes for $a=a_{M}$ [Fig. 25(d)]. Beyond the value $a_{M}$, just one saddle-node bifurcation remains, and its critical value increases with $a$ (see Fig. 25 at $a=0.68$ and $a=0.8$ ). When both saddle nodes are present, in the interval $d \in\left[0 ; d_{f 2}\right]$, there is a bistability between $s_{a}^{+}$and $s_{a}^{-}$. For $d$ between the two critical values $d_{f 2}$ and $d_{f 1}$, the dynamics converges to the remaining stable branch $s_{a}$. Finally, beyond the largest value of the saddle node the intermittent transport emerges. The transport has the same properties as previously, but a noteworthy behavior arises when the critical values are almost equal for $a=0.65\left(d_{f 1} \simeq d_{f 2} \simeq 0.05\right)$ [Fig. 25(c)]. Indeed, the Poincaré map of the dynamics displays a regular staircase with two different plateaux corresponding to the two periodic solutions that disappeared at the saddle-node bifurcations.

The numerical simulations show that the intermittent drift attracts all the dynamics, and it is still stable even for $d$ far from the onset. In addition, the transport does not change its direction and so the drift velocity $c$ remains negative. The drift velocity $|c(d)|$ is always an increasing function of $d$, hence the asymmetry parameter is also quantitatively relevant for the transport. The maximum drift velocity is not larger than 0.1 ; e.g., for $a=0.65, u_{m}=9$, and $d=0.4$, one obtains $c=-0.0654$, which is still small compared to that of the synchronized transport. It is noteworthy that the drift remains for large drag, at least for $\gamma=500$ as shown by the loci of 


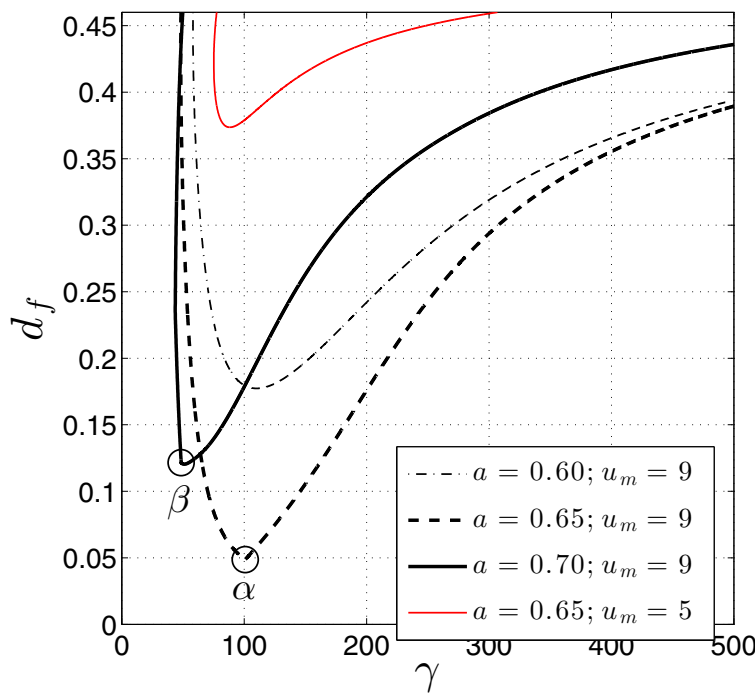

FIG. 28. Loci of intermittent bifurcation in the plane $\left(\gamma, d_{f}\right)$ for different values of the parameters $a$ and $u_{m}$. The upper domain bounded by each curve is the intermittent transport domain while in the lower domain the dynamics converges to a 1-periodic solution. The circles indicate corners of the curves discussed in the text.

bifurcation points in the $\left(\gamma, d_{f}\right)$ plane (Fig. 28). Hence, this transport may be relevant for microparticles. Beyond 500, the critical asymmetry parameter $d_{f}(\gamma)$ is larger than 0.4 , so this result is less relevant for our model. Owing to the intersection of loci of the two fold bifurcations displayed in Fig. 25(c), the curves corresponding to $a=0.65$ and $a=0.7$ have sharp corners $\alpha$ and $\beta$, respectively, which are the intersections of two loci of saddle nodes. Note that this intermittent transport does not exist for $\gamma$ smaller than 50; thus in contrast to the synchronized transport, this transport occurs for large drag. A transport solution is also found in the same direction for smaller characteristic velocities. However, its existence domain shrinks: if $u_{m}=5$ the range of intermittent bifurcation is $\gamma \in[80 ; 120]$ if we consider only $d<0.4$ (Fig. 28). For $\gamma=120$ the critical asymmetry parameter $d$ is larger than 0.4 , thus lager $\gamma$ values are not relevant.

The intermittent transport is always in the negative direction for $d>0$, and then, according to Sec. II B, the transport is in the positive direction for $d<0$. Therefore, the asymmetric shape of the pore profile induces the ratchet effect and determines its direction as for the mechanical pawl.

This slow drift is reminiscent of the description of the drift ratchet experiment and the result of the drift ratchet model. We therefore call this transport mechanism the deterministic drift ratchet.

\section{INTERPRETATION OF TRANSPORT TRANSITIONS}

In the following, we relate the transitions responsible for transport to the ratchet problem discussed in the literature and to the more general framework of dynamical systems.

\section{A. Transition of the deterministic drift ratchet}

The stroboscopic particle motion presented in Figs. 26 is very reminiscent of the phase slip addressed by Pikovsky et al.

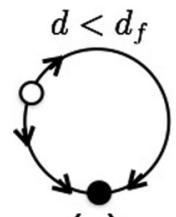

(a)

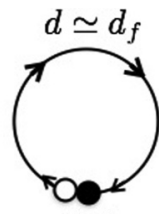

(b)

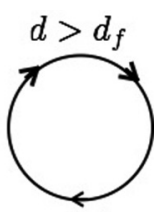

(c)
FIG. 29. Poincare section on the $S^{1}$ circle of the particle position $2 \pi x_{n}$ during the transition of the deterministic drift ratchet. (a) Full (empty) circles designate the 1-periodic stable (unstable) solution for $d<d_{f}$. Arrows on the circle indicate the direction of the heteroclinic connections between periodic solutions. The dynamics converges to the stable periodic solution. (b) At the onset $d=d_{f}$, unstable and stable mean positions coincide and only a homoclinic connection remains. (c) When $d>d_{f}$ the periodic solutions vanish and the dynamics leads to a rotation which corresponds to the particle drift.

$[26,62]$ and characterized by epochs of nearly constant phase intermingled with $2 \pi$ slips (see, e.g., Fig. 2 of Ref. [62]). Such a behavior is known as a loss of synchronization and can be found for many oscillators with a small forcing amplitude such as the Rössler oscillator [63]. However, this synchronization transition refers to the effect of a small external periodic force on a self-sustained oscillator or on a rotator [64]. The micropumping problem does not correspond to this framework: the periodic force is not small and without periodic pumping there is no motion; finally we do not consider a bias that could have played the role of the rotator [56]. Even so, the one-dimensional model Eq. (20) of the particle position is similar to the phase dynamics. Introducing the mean value of the function $g$, noted $-v$, and taking into account the properties of $g$, Eq. (20) reads

$$
x_{n+1}=x_{n}-v+\epsilon q\left(x_{n}\right),
$$

where $\epsilon=1 / \gamma, q$ is 1-periodic, and $v$ is positive. Note that $v$ is the distance from the curve of the return map to the bisectrix; it therefore measures the distance to the threshold $d-d_{c}$. By substituting $2 \pi x_{n}$ by the phase $\phi_{n}$, Eq. (21) becomes the phase dynamics approximation for synchronization of periodic oscillators by a weak periodic external action and with weak nonlinear oscillations [62]. Note that if $q(\phi)=\sin (\phi)$ then it is the well-known Adler equation [65]. In the following, we recall the properties of the phase dynamics presented in Ref. [26], which are clearly similar to the particle dynamics of Sec. IV. If the phase $\phi$ is represented by an angle in the phase plane, then the dynamics occurs on a circle, called the circle map. This circle is shown for the $2 \pi x_{n}$ dynamics for different steps of the drift transition in Fig. 29. On the circle, two regimes of the phase dynamics are possible, namely periodic or quasiperiodic. The periodic state corresponds to the existence of a pair of saddle fixed points on the circle map [Fig. 29(a)]. Note that more than two periodic solutions may exist but they always appear in pairs of saddle points [see Fig. 25(c)]. The loss of periodicity (desynchronization) arises from the saddle-node bifurcation [Fig. 29(b)]. Then the dynamics is characterized by a single parameter called the rotation number or beat frequency, which is equivalent to the particle drift velocity [Fig. 29(c)]. It is known that the rotation number does not depend on the initial position (see 
Ref. [66]) as we prove in the Appendix B for our particular case. Finally near the threshold, the rotation number has a square-root dependence $\sqrt{v-v_{c}}$. The difference $v-v_{c}$ is often called the frequency detuning [67]. In conclusion, the particle drift of small inertia is equivalent to the phase drift of an oscillator with weak forcing and the detuning parameter is the asymmetry $d$.

The slow intermittent particle transport of Sec. IV has been reported in a (stochastic) rocking ratchet for overdamped particles [32]. A bias plays the role of the depinning force leading to a particle transport with the same kind of quasiperiodic dynamics. In the deterministic case, the depinning transition is generally related to a global bifurcation as observed for the transport of attracting colloidal particles in Ref. [6] or for the depinning of drops on substrates with a periodic array of defects $[47,68]$. In the latter case, one of the depinning transitions is a SNIPer bifurcation (saddle-node infinite period), which involves at a saddle node a homoclinic loop as in our case [Fig. 29(b)]. It follows the same power law for the transport velocity near the onset. However, the resulting dynamics is periodic for the drop depinning while it is only quasiperiodic in our case. For the deterministic drift ratchet, it is due to the existence of two frequencies generically not commensurable in the dynamics: the pumping frequency and the rotation number.

\section{B. Transitions at moderate drag}

For moderate drag, the emergence of the periodic transport solution at a saddle node is also related to synchronization. Far enough away from the onset, there is a pair of saddles of periodic solution, and as previously the unstable manifold is connected to the stable periodic solution, which constitutes the invariant curve [Fig. 29(a)]. However, approaching the saddlenode bifurcation, the dynamics can no longer be reduced to the $S^{1}$ circle map. Instead, the dynamics $\left(\bar{x}_{n}, v_{n}\right) \in S^{1} \times \mathbb{R}$ tends asymptotically to an invariant annulus map if we represent the dynamics in polar coordinates $(\rho, \theta)$ such that $\rho_{n}=v_{n}+3 u_{m}$ and $\theta_{n}=2 \pi x_{n}$. The bounded character of $\rho_{n}$ ensues from the fact that the particle velocity is bounded by the fluid velocity. Such a map is similar to periodic oscillators with a moderate forcing where the amplitude of the oscillation corresponds to $\rho_{n}$ and the phase to $\theta_{n}$. In this framework, there are two scenarios of invariant curve destruction during the desynchronization summarized in Ref. [26] and described in greater detail in Refs. [69,70]. In the first scenario, the curve is no longer $C^{1}$ in the vicinity of the stable fixed point because of folds. In the second scenario, the unstable manifold also presents a fold, but additionally, it crosses the stable manifold, creating an homoclinic structure [55] and implying the existence of a nonattracting chaotic set usually called a repeller. In both scenarios beyond the saddle-node bifurcation the intermittency occurs as for weak driving. However, the lengths of the plateaux are no longer regular and are not determined solely by the rotation number. That is what we have observed near the onsets of synchronized transport. The figures show clearly that the velocity fluctuates and that it is not possible to define a clear limit independent of the initial condition. The second scenario seems to correspond to the dynamics near the transitions $\gamma_{f \ell}^{+}$and $\gamma_{f \ell}^{-}$. In particular, it corroborates the existence of a repeller near the threshold and explains the persistency of long chaotic dynamics even close to the saddle node (see Fig. 20). The second scenario quantitatively differs from the first scenario by a jump of the drift velocity during the transition.

Inside the existence domain, the periodic doubling cascade of the periodic transport can be interpreted as a destruction of the invariant curve in the generic problem of a driven oscillator too. Because the eigenvalues are complex, the period doubling of the stable fixed point breaks the circle map. The period-doubling cascade leads to a strange attractor appearing in the vicinity of the initial stable fixed point. The rotation still exists and remains the same till the crisis unlocks the transport velocity as we have shown.

The link between synchronization and periodic transport, for underdamped ratchets, was already interpreted as phase locking in the early 2000s [22] and explains why the drift velocity is commensurable [56]. In recent years, a proliferation of research focused on phase synchronization in the framework of coupled chaotic ratchets [71-74]. Nonetheless, the issue of the emergence of transport has been poorly addressed. In Ref. [23], the authors associated the existence of transport to the synchronization transition: "We showed that the occurrence of a net motion in the system is always related to phase locked dynamics" (see the conclusion of Ref. [23]). Our analysis shows that there is a small gap between the onset of the (phase unlocked) transport and the phase locked transport. According to the numerical simulation, synchronization optimizes only the drift velocity. The onset of unbounded dynamics is associated to a merging crisis (symmetric case) or a widening crisis (asymmetric case), which are well-known in chaos theory, especially as an intermittency mechanism [75,76]. However, to our knowledge, this onset has not been identified or mentioned in the ratchet framework. Moreover, even though the emergence of periodic transport at a saddle node was well established in Ref. [24], it is difficult to be detected using time integration only since this transition boundary may involve long chaotic transients as we observed close to the onsets $\gamma_{f \ell}^{-}$and $\gamma_{f \ell}^{+}$. Moreover, the stability range near the saddle node is often pointlike as shown by the bifurcation diagrams Figs. 12 and 18 and notably the existence domain (Fig. 14). Such stability domains with fine filaments are present in Fig. 1 of Ref. [24], but the study focused on the interior of the existence domain where the stability range is bounded by crises due to a doubling period route to chaos of the synchronized transport. Therefore the existence onset identified only via time integration may be confused with one of these crises inside the existence domain.

\section{CONCLUSION}

In this paper we have examined a nonlinear ODE as a model for the dynamics of particles confined in a periodic channel and dragged in a creeping flow. The particle drag force varies temporally and spatially as a standing wave. These variations constitute what we called the ratchet flow. Assuming the problem is axisymmetric, we obtain a second order differential equation. The equation differs slightly from the ratchet models usually employed in the literature. However, it possesses the main characteristics, namely, the time and spatial shift periods. 
According to Figs 14 and 28, particle transport exists for two orders of magnitude of the $\gamma$ drag: around 10 and more surprisingly around 100 through 500. In each of these ranges a specific bifurcation scenario occurs leading to transport, notably the role of the parity symmetry $\mathcal{S}$ differs. (1) When $\gamma$ is about 10 (Sec. III), spontaneous symmetry breaking leads to an unbounded dynamics and possible unidirectional transport even in the symmetric case. Obviously, we need asymmetry to obtain a net current in the statistical sense. (2) In contrast, the deterministic drift ratchet $(\gamma \sim 100)$ in Sec. IV is induced by the asymmetry, i.e., by a forced symmetry breaking. It is remarkable that even if the flow is slightly asymmetric $(d \sim 0.05)$, this transition may occur.

We retrace the two bifurcation scenarios starting from the 1-periodic solutions $s_{0}$ and $s_{m}$. Let us recall the main steps for each scenario:

(1) For a moderate drag of about 10, the emergence of transport is a consequence of spontaneous symmetry breaking, crisis and synchronization phenomena and the asymmetry can be interpreted as a perturbation of the symmetric case. So we considered the symmetric pore distribution leading to a problem invariant by the $\mathcal{S}$ symmetry for which $s_{0}$ and $s_{m}$ are symmetric solutions. By increasing the characteristic velocity $u_{m}$, the amplitude of oscillation increases and a pitchfork bifurcation leads to the asymmetric $s_{a}$ branch. The latter evolves a periodic doubling cascade inducing a bounded chaotic dynamics. By varying slightly the bifurcation parameter, the strange attractor collides via crisis with its neighbors which are copies shifted by one pore length. Then, the spatial shift symmetry is a necessary ingredient of the unbounded dynamics. In the vicinity of this crisis a saddle-node bifurcation marks the existence of a periodic and stable transport even in the symmetric case. This bifurcation can be interpreted as a synchronization phenomenon as for a self-sustained oscillator with moderate periodic driving (Sec. V). The two main kinds of intermittency observed near the saddle node are part of the two universal synchronization transitions described in Ref. [26]. Periodic transport exists in a range bounded by the saddle nodes. Because of the $\mathcal{S}$ symmetry, opposite transport solutions coexist. Inside its existence domain, a period-doubling phenomenon followed by a crisis occurs. Thereafter, the transport velocity is no longer locked and a chaotic and unbounded dynamics occurs.

The break of parity symmetry when $d \neq 0$ has two main consequences. First, the unbounded chaotic dynamics occurring after the crisis now displays a net current. Thus, the transport appears at the crisis of bounded strange attractors and not at the synchronization transition as suggested in the literature (see, e.g., Ref. [23]). Second, the existence domains of periodic transport are shifted and current reversals arise between two stability windows of opposite transports. Therefore, current reversal is a generic phenomenon of the slightly asymmetric ratchet problem involving synchronized transport. As explained in Sec. V, such a description of the transitions from periodic solution to synchronized transport has not yet been clearly discussed in the literature. The emergence of synchronized dynamics is not the threshold of the transport but rather corresponds to a transport optimisation since it is the maximum drift velocity.
(2) For the deterministic drift ratchet, the inertia of the particle is too small ( $\gamma$ about 100) to allow a perioddoubling phenomenon and chaotic dynamics. However, the periodic solutions $s_{0}, s_{m}$, or $s_{a}$ may collapse at a saddle-node bifurcation. This transition occurs only in the asymmetric case, since in the symmetric case the solutions have different mean positions. Beyond this saddle node a slow drift is shown by time integration. It increases with the asymmetry $d$ but remains smaller than 0.1: drift by one pore length needs at least 10 temporal periods. This transition is analogous to a depinning transition with a bias. In particular, the drift velocity vanishes as the square root of the threshold distance as for the SNIPer transition. The originality here is that there is no bias, only the flow asymmetry $d$ plays this role. In a more general framework, the particle drift is similar to the phase slip that occurs via a loss of synchronization of a self-sustained oscillator with a weak periodic driving. It follows that the slow drift is quasiperiodic and that the drift velocity is unique. Moreover the sign of $d$ determines the transport direction, and then a current reversal occurs by changing its sign. This kind of transport and current reversal induced by asymmetry is similar to the results of Refs. [21,30] for a rocking ratchet with biharmonic temporal forcing. The parameter $d$ plays the role of the phase difference in the temporal forcing. However, the drift velocity does not display the sinusoidal-like function or the more general function proposed in Ref. [31]. The different nature of the asymmetry (spatial in our case) and the particular form of $u_{0}$ which contains an infinity of harmonics may explain this difference. Anyway, this previous article cannot explain the transport mechanism and its emergence.

The numerical bifurcation analysis has shed new light on the classical single particle ratchet problem. The role of symmetry enables us not only to classify the types of transport mechanisms but also the two kinds of current reversal. For nearly overdamped particles, the drift ratchet is due to the flow asymmetry, and then asymmetry forces the direction, i.e., in our case the sign of the $d$ parameter. For smaller drag, the current reversal is due to the coexistence of opposite solutions in a symmetric case and any small break of this symmetry provides current reversal scenarios by varying one of the problem parameters such as the frequency or the amplitude of the forcing. We have clarified the role of the synchronization phenomenon in the ratchet effect. For an asymmetric flow profile (ratchet), synchronization is not responsible for the effective transport. Either it only optimizes the transport (Sec. III B) or on the contrary the loss of synchronization allows the transport (Sec. IV).

We also believe that in order to better understand the role of a weak noise on the dynamics, knowledge of the bifurcation diagrams is crucial. Indeed, the transports found are reminiscent of the dynamics of noisy ratchets as in Ref. [77]. The vicinity of the shifted period-two orbits described in Sec. III A 4 may be a possible explanation for the triggering of the onset of transport found in this paper. For large drag, the dynamics is also similar to the noisy overdamped case $[32,78]$. Therefore, the small particle acceleration seems to play a similar role to that of a small Brownian noise. Recently, in Ref. [79], it was shown how noise may trigger the phase slip, i.e., the drift in our context. 
One perspective of this study concerns the improvement of the model for an arbitrary particle radius which is especially relevant for drag coefficients $\gamma$ of about 10. In Refs. [36,37], it is shown that variations in the drag coefficient $\gamma(x)$ can be non-negligible when the particle radius is comparable to the minimum radius of the channel. A ratchet due to drag coefficient variations is known as a friction ratchet $[10,80]$. In a current project we therefore aim at performing a similar bifurcation analysis by combining flow and friction ratchets.

\section{ACKNOWLEDGMENTS}

This work has been initially supported by the DFG [SFB 486, project B13]. P.B. is grateful to P. Talkner for helpful discussions and for his carefully reading of early drafts. The authors wish also to thank the reviewers for their valuable comments and suggestions.

\section{APPENDIX A: SMALL AMPLITUDE DRIVING}

Assuming that the amplitude of the fluid is small compared to the pore length, we prove that the drift converges to a stable solution. Similar results are obtained by ponderomotive force; here, however, the particle drag is not negligible contrary to these studies. Let us now detail the proof.

The small amplitude assumption implies that the mean velocity $u_{m}$ is small, i.e., $u_{m} \ll 1$, which constitutes the small parameter in the following developments. We decompose the particle trajectory

$$
x(t)=x_{p}(t)+x_{d}(t)
$$

as the sum of a 1-periodic function $x_{p}$ (short time variation) and of a slow drift $x_{d}$. More precisely, we set the ansatz: $x_{p}=O\left(u_{m}\right), \dot{x}_{d}=O\left(u_{m}^{2}\right)$, and $\ddot{x}_{d}=O\left(u_{m}^{2}\right)$. Hence, during one period, we may assume $x_{d}(t)$ constant, and we have the following approximation:

$$
\begin{aligned}
x_{d}(t) & =x_{d}\left(t_{0}\right)+O\left(u_{m}^{2}\right) \simeq x_{d}^{0}, \\
u_{0}(x(t)) & =u_{0}\left(x_{p}(t)+x_{d}(t)\right) \\
& =u_{0 d}+u_{0 d}^{\prime} x_{p}+O\left(u_{m}^{3}\right)
\end{aligned}
$$

with $u_{0 d}=u_{0}\left[x_{d}\left(t_{0}\right)\right]$ and $u_{0 d}^{\prime}=u_{0}^{\prime}\left(x_{d}\right)=\frac{d u_{0}}{d x}\left[x_{d}\left(t_{0}\right)\right]$. By replacing $x(t)$ by its decomposition Eq. (A1) in the governing equation Eq. (4):

$$
\begin{aligned}
\ddot{x}_{d} & +\ddot{x}_{p}+\gamma \dot{x}_{d}+\gamma \dot{x}_{p} \\
& =\gamma\left(u_{0 d}+u_{0 d}^{\prime} x_{p}\right) \sin 2 \pi t+O\left(u_{m}^{3}\right) .
\end{aligned}
$$

We study this equation by focusing on two different scales: the short time where $x_{d}$ is constant and the long time scale by taking the mean value over one period, noted $\langle\cdot\rangle$. Thus, we obtain two equations:

$$
\begin{gathered}
\ddot{x}_{p}+\gamma \dot{x}_{p}=\gamma u_{0 d} \sin 2 \pi t+O\left(u_{m}^{2}\right) \\
\left\langle\ddot{x}_{d}\right\rangle+\gamma\left\langle\dot{x}_{d}\right\rangle=\gamma u_{0 d}^{\prime}<x_{p}(t) \sin 2 \pi t>+O\left(u_{m}^{3}\right) .
\end{gathered}
$$

The short time Eq. (A5) can be solved at leading order

$$
x_{p}(t)=-\frac{u_{d}}{2 \pi}\left[1+\left(\frac{2 \pi}{\gamma}\right)^{2}\right]^{-1 / 2} \sin (2 \pi t+\varphi)+O\left(u_{m}^{2}\right)
$$

with the phase $\varphi \in] 0, \pi / 2[$ such that $\tan \varphi=\gamma /(2 \pi)$. Replacing this solution in Eq. (A6), the governing equation of the slow drift is at leading order

$$
\ddot{x}_{d}+\gamma \dot{x}_{d}=-\gamma \alpha u_{0}\left(x_{d}\right) u_{0}^{\prime}\left(x_{d}\right),
$$

with $\alpha=\frac{1}{4 \pi}\left(1+(2 \pi / \gamma)^{2}\right)^{-1 / 2} \cos \varphi>0$ and $x_{d}$ is identified with its mean value. The drift vanishes when $u_{0}^{\prime}=0$, i.e., at the extrema of the velocity field. A standard stability analysis shows that at a local minimum of $u_{0}$, the equilibrium is stable while at a maximum it is a saddle, in particular, it is unstable. In conclusion, for sufficiently small driving, we expect the particles to collect at the velocity minima.

\section{APPENDIX B: REGULAR INTERMITTENT TRANSPORT}

The intermittent transport for large drag $\gamma$ displays a very regular aspect. Let us prove that the two-dimensional map $x_{n+1}=f\left(x_{n}\right)=x_{n}+g\left(x_{n}\right)$ with the four assumptions (1) $g$ is 1-periodic, (2) $g$ is small, (3) $\left|g^{\prime}\right|$ is not large $(<1)$, and (4) $g$ is strictly negative can explain this behavior. The two-dimensional aspect of the dynamics is discussed later.

We construct the map $x_{n}$ starting from $x_{0}$. Because $g$ is strictly negative, $x_{n}$ is strictly decreasing. We call $N$ the smallest iteration number such that $x_{N} \leqslant$ $x_{0}-1$ (Fig. 30). Assumption 3 implies that $f$ is a strictly increasing function. Then $x_{0}-1<x_{N-1}$ implies that $x_{1}-1<x_{N}$.

Now consider the map $y_{n}$ starting from $y_{0}$ in the interval ]$\left.x_{1} ; x_{0}\right]$. We seek the minimal iteration number $M$ such that $y_{M}<x_{0}-1$. Because $f$ is strictly decreasing, then $x_{n+1}<y_{n} \leqslant x_{n}$ for all $n$. For instance, $x_{N}<y_{N-1} \leqslant x_{N-1}$. The range $\left[x_{N}, x_{N-1}\right]$ contains the point $y_{0}-1$ [Fig. 30], and there are two cases depending on whether $y_{N-1}$ is inferior to $y_{0}-1$ or not. If $y_{N-1} \leqslant y_{0}-1$, then $M=N-1$ is the iteration number. Otherwise $x_{1}-1<y_{N} \leqslant x_{N} \leqslant y_{0}-1$ and then $M=N$ (case considered in Fig. 30).

Therefore the map $y_{n}$ modulo 1 passes in the interval $\left.] x_{1}, y_{0}\right]$ every $N-1$ or $N$ iterations. Because $y_{0}$ is unspecified all dynamics have this quasiperiodic behavior. Furthermore, if $y_{0}$ is near the tangent bifurcation, then $] x_{1}, y_{0}$ ] is small and then the dynamics appears regular.

The previous proof does not hold if one considers the twodimensional discrete dynamical system taking velocity into

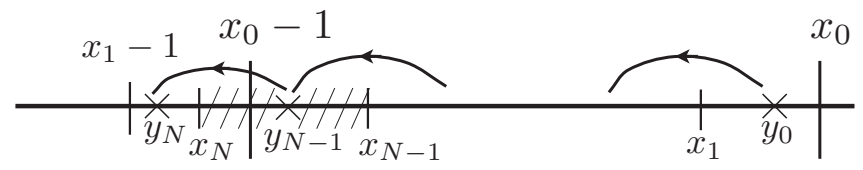

FIG. 30. Scheme of the $\left(x_{n}\right)$ and $\left(y_{n}\right)$ maps. The hatched segment is the range of possible values of $y_{N-1}$. If $x_{0}-1<y_{N-1}$ as in this scheme then $x_{1}-1<y_{N}<x_{N}$. 
account. According to the numerical simulations, it acts as a small perturbation to the previous process. Approaching the bifurcation point, the intervals $\left[x_{n}, x_{n+1}\right]$ vanish. Even a small perturbation of this map may then have a great influence on the number of iterations. This is what was observed when $d$ is very close to $d_{f}$. However, the variation of iterations is smaller compared to the mean iteration number and the regular aspect remains.
[1] M. Kostur, M. Schindler, P. Talkner, and P. Hänggi, Phys. Rev. Lett. 96, 014502 (2006).

[2] A. Russom, A. K. Gupta, S. Nagrath, D. D. Carlo, J. F. Edd, and M. Toner, New J. Phys. 11, 075025 (2009).

[3] A. Bhagat, S. Kuntaegowdanahalli, and I. Papautsky, Microfluidics Nanofluidics 7, 217 (2009).

[4] D. Di Carlo, J. F. Edd, K. J. Humphry, H. A. Stone, and M. Toner, Phys. Rev. Lett. 102, 094503 (2009).

[5] H. Nieuwstadt, R. Seda, D. Li, J. Fowlkes, and J. Bull, Biomed. Microdevices 13, 97 (2011).

[6] A. Pototsky, A. J. Archer, S. E. Savel'ev, U. Thiele, and F. Marchesoni, Phys. Rev. E 83, 061401 (2011).

[7] J. Rousselet, L. Salome, A. Ajdari, and J. Prostt, Nature (London) 370, 446 (1994).

[8] S. Matthias and F. Müller, Nature (London) 424, 53 (2003).

[9] C. Kettner, P. Reimann, P. Hänggi, and F. Müller, Phys. Rev. E 61, 312 (2000).

[10] P. Reimann, Phys. Rep. 361, 57 (2002).

[11] P. Hänggi, F. Marchesoni, and F. Nori, Ann. der Phys. 14, 51 (2005).

[12] P. Hänggi and F. Marchesoni, Rev. Mod. Phys. 81, 387 (2009).

[13] K. Mathwig, F. Müller, and U. Gösele, New J. Phys. 13, 033038 (2011).

[14] P. Blondeaux, G. Vittori, A. Bruschi, F. Lalli, and V. Pesarino, J. Fluid Mech. 697, 115 (2012).

[15] F. Mugele, A. Staicu, R. Bakker, and D. van den Ende, Lab Chip 11, 2011 (2011).

[16] J. A. Y. C. McWilliams, P. P. Sullivan, and C.-H. Moeng, J. Fluid Mech. 334, 1 (1997).

[17] F. Santamaria, G. Boffetta, M. M. Afonso, A. Mazzino, M. Onorato, and D. Pugliese, Europhys. Lett. 102, 14003 (2013).

[18] S. Flach, O. Yevtushenko, and Y. Zolotaryuk, Phys. Rev. Lett. 84, 2358 (2000).

[19] O. Yevtushenko, S. Flach, Y. Zolotaryuk, and A. A. Ovchinnikov, Europhys. Lett. 54, 141 (2001).

[20] P. Reimann, Phys. Rev. Lett. 86, 4992 (2001).

[21] D. Cubero, V. Lebedev, and F. Renzoni, Phys. Rev. E 82, 041116 (2010).

[22] J. L. Mateos, Phys. Rev. Lett. 84, 258 (2000).

[23] M. Barbi and M. Salerno, Phys. Rev. E 62, 1988 (2000).

[24] D. Speer, R. Eichhorn, and P. Reimann, Phys. Rev. E 76, 051110 (2007).

[25] P. S. Landa, Nonlinear Oscillations and Waves in Dynamical Systems (Kluwer Academic Publishers, Dordrecht, 1996).

[26] A. Pitkovsky, M. Rosenblum, and J. Kurths, Synchronization. A Universal Concept in Nonlinear Sciences (Cambridge University Press, Cambridge, 2001).

[27] J. L. Mateos, Physica D 168-169, 205 (2002).

[28] L. Du and D. Mei, Phys. Rev. E 85, 011148 (2012).

[29] N. R. Quintero, J. A. Cuesta, and R. Alvarez-Nodarse, Phys. Rev. E 81, 030102 (2010).
[30] A. Wickenbrock, D. Cubero, N. A. Abdul Wahab, P. Phoonthong, and F. Renzoni, Phys. Rev. E 84, 021127 (2011).

[31] J. A. Cuesta, N. R. Quintero, and R. Alvarez-Nodarse, Phys. Rev. X 3, 041014 (2013).

[32] K. Lee, J. Korean Phys. Soc. 60, 1845 (2012).

[33] H. A. Dijkstra1, F. W. Wubs, A. K. Cliffe, E. Doedel, I. F. Dragomirescu, B. Eckhardt, A. Y. Gelfgat, A. L. Hazel, V. Lucarini, A. G. Salinger, E. T. Phipps, J. Sanchez-Umbria, H. Schuttelaars, L. S. Tuckerman, and U. Thiele, Commun. Comput. Phys. 15, 1 (2014).

[34] H.-J. Bungartz, M. Mehl, T. Neckel, and T. Weinzierl, Comput. Mech. 46, 103 (2010).

[35] J. Happel and B. Bryne, Ind. Eng. Chem. 56, 1181 (1954).

[36] M. Makhoul, P. Beltrame, and M. Joelson, in Topical Problems of Fluid Mechanics, edited by D. Simurda and T. Bodnar (CTU, Prague Fac. Mech. Eng., 2015), pp. 137-144.

[37] M. Makhoul, P. Beltrame, and M. Joelson, Intl. J. Mech. 9, 260 (2015).

[38] H. Brenner, J. Fluid Mech. 18, 144 (1964).

[39] M. Makhoul, P. Beltrame, and M. Joelson, in Advances in Mathematics and Statistical Sciences, edited by N. E. Mastorakis, A. Ding, and M. V. Shitikova (WSEAS Press, Dubai, United Arab Emirates, 2015), pp. 40-47.

[40] E. Doedel, R. Paffenroth, A. Champneys, T. Fairgrieve, Y. Kuznetsov, B. Sandstede, and X. Wang, AUTO 2000: Continuation and Bifurcation Software for Ordinary Differential Equations (with HomCont), Tech. Rep. (Caltech, Pasadena, 2001).

[41] E. J. Doedl, Numerical Continuation Methods for Dynamical Systems, edited by B. Krauskopf, H. M. Osinga, and J. GalánVioque (Springer, Netherlands, 2007), pp. 1-49.

[42] J. C. Alexander, E. J. Doedel, and H. J. Othmer, SIAM J. Appl. Math. 50, 1373 (1990).

[43] E. Doedel, W. Govaerts, and Y. Kuznetsov, SIAM J. Numer. Anal. 41, 401 (2003).

[44] Y. A. Kuznetsov, in Elements of Applied Bifurcation Theory, edited by S. S. Antman, J. E. Marsden, and L. Sirovich, Applied Mathematical Sciences, Vol. 112 (Springer, New York, 2004), pp. 77-115.

[45] P. L. Similon and A. N. Kaufman, Phys. Rev. Lett. 53, 1061 (1984).

[46] B. W. J. McNeil and N. R. Thompson, Nat. Photon. 4, 814 (2010).

[47] U. Thiele and E. Knobloch, New J. Phys. 8, 313 (2006).

[48] P. Beltrame and U. Thiele, SIAM J. Appl. Dyn. Syst. 9, 484 (2010).

[49] J. Burke and E. Knobloch, Phys. Lett. A 360, 681 (2007).

[50] U. Thiele, A. J. Archer, M. J. Robbins, H. Gomez, and E. Knobloch, Phys. Rev. E 87, 042915 (2013).

[51] J. Testa, J. Pérez, and C. Jeffries, Phys. Rev. Lett. 48, 714 (1982).

[52] J. W. Swift and K. Wiesenfeld, Phys. Rev. Lett. 52, 705 (1984). 
[53] C. Tresser and P. Coullet, C. R. Acad. Sc. Paris A 287, 577 (1978).

[54] M. J. Feigenbaum, J. Stat. Phys. 21, 669 (1979).

[55] C. Grebogi, E. Ott, and J. A. Yorke, Phys. Rev. Lett. 57, 1284 (1986).

[56] J. L. Mateos and F. R. Alatriste, Chaos 18, 043125 (2008).

[57] W.-S. Son, I. Kim, Y.-J. Park, and C.-M. Kim, Phys. Rev. E 68, 067201 (2003).

[58] P. Manneville and Y. Pomeau, Physica D 1, 219 (1980).

[59] Y. Pomeau and P. Manneville, Commun. Math. Phys. 74, 189 (1980).

[60] V. Franceschini, C. Gilbert, and Z. Zheng, Nonlinearity 6, 251 (1993).

[61] B. Coombes, H. Kocak, and K. J. Palmer, Computation of Long Periodic Orbits in Chaotic Dynamical Systems, CADSEM Report, Centre for Applied Dynamical Systems and Environmental Modelling (Deakin University, Melbourne, 1996).

[62] A. Pikovsky, M. Rosenblum, and J. Kurths, Int. J. Bifurcation Chaos 10, 2291 (2000).

[63] K. J. Lee, Y. Kwak, and T. K. Lim, Phys. Rev. Lett. 81, 321 (1998).

[64] F. R. Alatriste and J. L. Mateos, Physica A 372, 263 (2006).

[65] R. Adler, Proc. IEEE 61, 1380 (1973).

[66] A. Katok and B. Hasselblatt, Introduction to the Modern Theory of Dynamical Systems (Cambridge University Press, Cambridge, 1996).
[67] C. Brandt, O. Grulke, T. Klinger, J. Negrete, G. Bousselin, F. Brochard, G. Bonhomme, and S. Oldenbürger, Phys. Rev. E 84, 056405 (2011).

[68] P. Beltrame, E. Knobloch, P. Hänggi, and U. Thiele, Phys. Rev. E 83, 016305 (2011).

[69] D. Aronson, M. Chory, G. Hall, and R. McGehee, Commun. Math. Phys. 83, 303 (1982).

[70] V. S. Afraimovich and L. P. Shilnikov, Amer. Math Soc. Transl. 149, 201 (1991).

[71] U. E. Vincent, A. N. Njah, O. Akinlade, and A. R. T. Solarin, Chaos 14, 1018 (2004).

[72] S. Denisov, Physica A 377, 429 (2007).

[73] J. L. Mateos and F. Alatriste, Chem. Phys. 375, 464 (2010).

[74] D. Cubero and F. Renzoni, Phys. Rev. E 86, 056201 (2012).

[75] M. Sauer, J. Leonardy, and F. Kaiser, Chaos Solitons Fractals 4, 1461 (1994).

[76] M. Münkel and F. Kaiser, Physica D 98, 156 (1996).

[77] L. Machura, M. Kostur, P. Talkner, J. Luczka, and P. Hanggi, Phys. Rev. Lett. 98, 040601 (2007).

[78] A. K. Lee, J.-R. Lee, and K. Lee, Physica B 407, 4298 (2012).

[79] Z. B. Guo and P. H. Diamond, Phys. Rev. Lett. 114, 145002 (2015).

[80] R. H. Luchsinger, Phys. Rev. E 62, 272 (2000). 